TRANSACTIONS OF THE

AMERICAN MATHEMATICAL SOCIETY

Volume 360, Number 6, June 2008, Pages 3345-3364

S 0002-9947(08)04445-0

Article electronically published on January 11, 2008

\title{
STOCHASTIC EQUILIBRIA IN VON NEUMANN-GALE DYNAMICAL SYSTEMS
}

\author{
IGOR V. EVSTIGNEEV AND KLAUS REINER SCHENK-HOPPÉ
}

\begin{abstract}
This paper examines a class of random dynamical systems related to the classical von Neumann and Gale models of economic dynamics. Such systems are defined in terms of multivalued operators in spaces of random vectors, possessing certain properties of convexity and homogeneity. We establish a general existence theorem for equilibrium, which holds under conditions analogous to the standard deterministic ones. Our results answer questions that remained open for more than three decades.
\end{abstract}

\section{INTRODUCTION}

Von Neumann-Gale dynamical systems are defined in terms of multivalued operators that possess certain properties of convexity and homogeneity. These operators assign to each element of a given cone a convex subset of the cone describing possible one-step transitions from one state of the system to another. The classical, deterministic theory of such dynamics was originally aimed at the modeling of economic growth (von Neumann [29], Gale [14]). First attempts to build a stochastic generalization of this theory were undertaken in the 1970s by Dynkin $[6,7,8]$, Radner $[23,24]$ and others. However, the initial attack on the problem left many questions unanswered. Substantial progress was made only in the 1990s; see the survey in [11].

It has recently been observed [4] that stochastic analogues of von NeumannGale systems provide a natural and convenient framework for financial modeling (asset pricing and hedging under transaction costs). This observation gave a new momentum to studies in the field and posed new interesting questions. It also revived interest in old unsolved problems.

In spite of the current progress achieved, a substantial gap remained. The theory lacked quite satisfactory results on the existence of equilibrium in stochastic von Neumann-Gale systems. The results available established the existence of equilibrium under certain conditions which also guaranteed its stability. These conditions seemed to be too restrictive in the context of economic models and did not cover a number of new examples arising in financial applications. Furthermore, the conditions were substantially distinct from those conventionally imposed in the deterministic case. In this paper, we fill this gap by establishing the existence of equilibrium under assumptions fully analogous to the standard deterministic ones.

Received by the editors July 5, 2006 and, in revised form, October 27, 2006.

2000 Mathematics Subject Classification. Primary 37H99, 37H15; Secondary 91B62, 91B28.

Key words and phrases. Random dynamical systems, convex multivalued operators, von Neumann-Gale model, rapid paths, convex duality, stochastic equilibrium. 
The problem of obtaining a result of this kind was posed by E.B. Dynkin in the early 1970s. We are happy to provide (alas, only after three decades!) a solution to the problem. The result obtained is applicable to both old models - those related to economics - and new ones, coming from finance.

We analyze a stochastic version of a von Neumann-Gale dynamical system defined in terms of a stationary random process $s_{t}, t=0, \pm 1, \ldots$, with values in a measurable space $S$ and a closed convex cone $C\left(\ldots, s_{-1}, s_{0}\right) \subseteq \mathbb{R}_{+}^{n} \times \mathbb{R}_{+}^{n}$ depending measurably ${ }^{1}$ on $\left(\ldots, s_{-1}, s_{0}\right)$. We denote by $s^{t}:=\left(\ldots, s_{t-1}, s_{t}\right)$ the "history" of the process $\ldots, s_{0}, s_{1}, \ldots$ up to time $t$ and consider the class $Z_{t}$ of pairs $\left(x\left(s^{t-1}\right), y\left(s^{t}\right)\right)$ of essentially bounded measurable functions such that

$$
\left(x\left(s^{t-1}\right), y\left(s^{t}\right)\right) \in C\left(s^{t}\right)
$$

almost surely (a.s.). We put $Z_{t}(x):=\left\{y:(x, y) \in Z_{t}\right\}$. The operators $Z_{1}(\cdot), Z_{2}(\cdot), \ldots$ define the multivalued dynamical system we study. For each $t \geq 1$, the multivalued mapping $x \mapsto Z_{t}(x)$ describes the set of those random states $y\left(s^{t}\right)$ of the system which can be reached at time $t$ from the random state $x\left(s^{t-1}\right)$ at time $t-1$. The main characteristic feature of such dynamics is that the graphs $Z_{t}$ of the operators $Z_{t}(\cdot)$ are convex cones. It will be convenient to deal with the graphs $Z_{t}$, rather than with the operators $Z_{t}(\cdot)$ themselves (although these ways of presentation are of course equivalent).

A sequence of functions $x_{0}\left(s^{0}\right), x_{1}\left(s^{1}\right), \ldots$ is called a path (trajectory) in the dynamical system under consideration if

$$
\left(x_{t-1}, x_{t}\right) \in Z_{t}
$$

for all $t \geq 1$. The fact that $x_{t}\left(s^{t}\right)$ is a function of $s^{t}$ means that a random state of the system may depend on the past and the present of the underlying process $\left(s_{t}\right)$ but cannot depend on its future. We will be mainly interested in those trajectories that grow in a sense faster than others - we will call them "rapid". To define them, we will need an important auxiliary notion of a dual path. Put

$$
C^{\times}\left(s^{t}\right):=\left\{(p, q) \in \mathbb{R}_{+}^{n} \times \mathbb{R}_{+}^{n}: q b-p a \leq 0 \text { for all }(a, b) \in C\left(s^{t}\right)\right\}
$$

and denote by $Z_{t}^{\times}$the set of pairs $\left(p\left(s^{t}\right), q\left(s^{t}\right)\right)$ of measurable vector functions such that

$$
\left(p\left(s^{t}\right), q\left(s^{t}\right)\right) \in C^{\times}\left(s^{t}\right) \text { (a.s.), }
$$

$E\left|p\left(s^{t}\right)\right|<\infty$ and $E\left|q\left(s^{t}\right)\right|<\infty$. Here " $E$ " stands for the expectation with respect to the underlying probability $P$, and the norm $\left|\left(p^{1}, \ldots, p^{n}\right)\right|$ is defined as $\left|p^{1}\right|+\ldots+$ $\left|p^{n}\right|$. A dual path (dual trajectory) is a sequence $p_{1}\left(s^{1}\right), p_{2}\left(s^{2}\right), \ldots$ of non-negative measurable vector functions satisfying

$$
\left(p_{t}, E_{t} p_{t+1}\right) \in Z_{t}^{\times}, t=1,2, \ldots
$$

where $E_{t}(\cdot)=E\left(\cdot \mid s^{t}\right)$ is the conditional expectation given $s^{t}$. By virtue of $(1.2)$ and (1.3), $E_{t}\left(p_{t+1} y\right) \leq p_{t} x$ (a.s.) for any $(x, y) \in Z_{t}$. This inequality shows that for any path $x_{0}, x_{1}, \ldots$ the sequence of random variables $p_{1} x_{0}, p_{2} x_{1}, \ldots$ is a supermartingale with respect to the filtration in the underlying probability space generated by $s^{t}, t=1,2, \ldots$.

A dual path $p_{1}, p_{2}, \ldots$ is said to support a path $x_{0}, x_{1}, \ldots$ if

$$
p_{t+1} x_{t}=1 \text { (a.s.) }
$$

\footnotetext{
${ }^{1}$ A closed set $C(s)$ is said to depend measurably on the parameter $s$ if for each point $x$ the distance between $x$ and $C(s)$ is a measurable function of $s$.
} 
for all $t \geq 0$. A trajectory is called rapid if there exists a dual trajectory supporting it. The term is motivated by the fact that

$$
\frac{E_{t}\left(p_{t+1} y_{t}\right)}{p_{t} y_{t-1}} \leq \frac{E_{t}\left(p_{t+1} x_{t}\right)}{p_{t} x_{t-1}}=1 \text { (a.s.) }
$$

for each path $y_{0}, y_{1}, \ldots$ with $p_{t} y_{t-1}>0$ (see (1.3) and (1.4)). This means that a rapid path $x_{0}, x_{1}, \ldots$ maximizes with probability one the conditional expectation of the growth rate at each time $t$, the maximum being equal to 1 . Growth rates are measured in terms of random linear functions $p_{t} a$. In the applications, vectors $x_{t}$ constituting trajectories can represent, e.g., commodity bundles or portfolios of assets; then $p_{t}$ are interpreted as prices.

A path $x_{0}, x_{1}, \ldots$ is called balanced if there exist essentially bounded measurable vector and scalar functions $x\left(s^{0}\right) \geq 0$ and $\lambda\left(s^{1}\right)>0$ such that

$$
x_{0}\left(s^{0}\right)=x\left(s^{0}\right), x_{t}\left(s^{t}\right)=\lambda\left(s^{t}\right) \ldots \lambda\left(s^{1}\right) x\left(s^{t}\right), t=1,2, \ldots,
$$

and

$$
\left|x\left(s^{0}\right)\right|=1 \text { (a.s.). }
$$

Such trajectories grow at a stationary rate and with stationary proportions defined by $\lambda\left(s^{t}\right)$ and $x\left(s^{t}\right)$, respectively. Clearly a pair of essentially bounded measurable functions $x\left(s^{0}\right) \geq 0$ and $\lambda\left(s^{1}\right)>0$ generates a balanced path if and only if condition (1.6) holds and

$$
\left(x\left(s^{0}\right), \lambda\left(s^{1}\right) x\left(s^{1}\right)\right) \in C\left(s^{1}\right) \text { (a.s.). }
$$

By virtue of stationarity of the process $\left(s_{t}\right)$, the functional $E \ln \lambda\left(s^{t}\right)$ (taking values in $[-\infty,+\infty))$ does not depend on $t$. A balanced path maximizing this functional is termed a von Neumann path.

A dual path $p_{1}, p_{2}, \ldots$ is called balanced if there exist measurable vector and scalar functions $p\left(s^{1}\right) \geq 0$ and $\lambda\left(s^{1}\right)>0$ such that

$$
p_{1}\left(s^{1}\right)=p\left(s^{1}\right), p_{t}\left(s^{t}\right)=\frac{p\left(s^{t}\right)}{\lambda\left(s^{t-1}\right) \ldots \lambda\left(s^{1}\right)}, t=2,3, \ldots,
$$

and

$$
E\left|p\left(s^{1}\right)\right|<\infty
$$

We have

and

$$
\left(p_{1}, E_{1} p_{2}\right)=\left(p\left(s^{1}\right), \frac{E_{1} p\left(s^{2}\right)}{\lambda\left(s^{1}\right)}\right)
$$

$$
\left(p_{t}, E_{t} p_{t+1}\right)=\left(\frac{p\left(s^{t}\right)}{\lambda\left(s^{t-1}\right) \ldots \lambda\left(s^{1}\right)}, \frac{E_{t} p\left(s^{t+1}\right)}{\lambda\left(s^{t}\right) \ldots \lambda\left(s^{1}\right)}\right), t=2,3, \ldots
$$

From this we can see that a pair of measurable functions $p\left(s^{1}\right) \geq 0$ and $\lambda\left(s^{1}\right)>0$ generates a balanced dual path if and only if

$$
\left(p\left(s^{1}\right), \frac{E_{1} p\left(s^{2}\right)}{\lambda\left(s^{1}\right)}\right) \in C^{\times}\left(s^{1}\right) \text { (a.s.) }
$$

and condition (1.9) holds. (Here we use the fact that if $E\left|p_{1}\right|<\infty$, then $E\left|p_{t}\right|<\infty$ for all $t$; this is so under the assumptions we impose below.)

A triplet of measurable functions $x\left(s^{0}\right) \geq 0, p\left(s^{1}\right) \geq 0, \lambda\left(s^{1}\right)>0$ forms a von Neumann equilibrium if the sequence $x_{0}, x_{1}, \ldots$ defined by (1.5) is a balanced path 
and the sequence $p_{1}, p_{2}, \ldots$ defined by (1.8) is a dual balanced path supporting it. The last condition holds if and only if

$$
p\left(s^{1}\right) x\left(s^{0}\right)=1 \text { (a.s.). }
$$

Therefore a triplet $x\left(s^{0}\right) \geq 0, p\left(s^{1}\right) \geq 0, \lambda\left(s^{1}\right)>0$ is an equilibrium if and only if the functions $x, p$ and $\lambda$ are measurable, $x$ and $\lambda$ are essentially bounded, and conditions $(1.6),(1.7),(1.9),(1.10)$ and (1.11) are fulfilled. It can be shown (see Proposition 3 below) that if $(x, p, \lambda)$ is an equilibrium, then the pair $(x, \lambda)$ generates a von Neumann path. Note that the latter is rapid because it is supported by the dual trajectory $p_{1}, p_{2}, \ldots$.

Consider for the moment the deterministic case, i.e. assume that $S$ consists of a single point, and suppose that

$$
C=\left\{(a, b) \in \mathbb{R}_{+}^{n} \times \mathbb{R}_{+}^{n}: b \leq H a\right\},
$$

where $H$ is a positive matrix (all inequalities between vectors are understood coordinatewise). Then $(x, p, \lambda)$ is a von Neumann equilibrium if and only if $\lambda$ is the Perron-Frobenius eigenvalue of $H$, and $x$ and $p$ are the Perron-Frobenius eigenvectors of $H$ and of the conjugate to $H$, respectively. In random systems defined in terms of positive matrices, $x, p$ and $\lambda$ are stochastic analogues of such eigenvectors and eigenvalues; see Evstigneev [9], Arnold, Demetrius and Gundlach [3], and Kifer [18].

Let us formulate the assumptions we impose on the dynamical system at hand. For each $s^{t}$, the cone $C\left(s^{t}\right)$ is supposed to satisfy the following conditions:

(C.1) For any $a \in \mathbb{R}_{+}^{n}$, the set $\left\{b:(a, b) \in C\left(s^{t}\right)\right\}$ is non-empty.

(C.2) The set $C\left(s^{t}\right)$ contains with every $(a, b)$ all $\left(a^{\prime}, b^{\prime}\right)$ such that $a^{\prime} \geq a$ and $0 \leq b^{\prime} \leq b$. $s^{t}$.

(C.3) There exists a constant $M$ such that $|b| \leq M|a|$ for all $(a, b) \in C\left(s^{t}\right)$ and

Clearly, it is sufficient to define $C\left(s^{t}\right)$ and impose conditions (C.1) - (C.3) for some particular $t$, say $t=0$. Then $C\left(s^{t}\right)$ will be defined and will satisfy conditions (C.1)-(C.3) for all integers $t$. The conditions imposed are quite standard in the theory under consideration (in the economic applications, property $(\mathbf{C . 2})$ is referred to as a "free disposal hypothesis").

We will also assume that the following requirement is fulfilled.

(C.4) There exist a constant $\gamma>0$ and an integer $l \geq 1$ such that for every $i=1, \ldots, n$ one can find measurable vector functions $y_{1, i}\left(s^{1}\right), \ldots, y_{l, i}\left(s^{l}\right)$ satisfying

$$
\left(e_{i}, y_{1, i}\right) \in Z_{1},\left(y_{1, i}, y_{2, i}\right) \in Z_{2}, \ldots,\left(y_{l-1, i}, y_{l, i}\right) \in Z_{l}, y_{l, i} \geq \gamma e .
$$

We write $e$ for the vector $(1, \ldots, 1) \in \mathbb{R}^{n}$, and we denote by $e_{i}$ the vector in $\mathbb{R}^{n}$ whose coordinates are all equal to zero except the $i$ th coordinate which is equal to 1 . According to (C.4), one can reach the strictly positive vector $\gamma e$ in $l$ steps starting from any of $e_{i}, i=1,2, \ldots, n$. It is known $[16,17]$ that even in the deterministic case an equilibrium might fail to exist if condition (C.4) does not hold.

One might wish to make the constants $M$ and $\gamma$ in (C.3) and (C.4) random (satisfying proper assumptions of integrability). Our methods, however, do not allow for that. Conditions (C.3) and (C.4), as they are stated above, guarantee the possibility of working within the framework of the duality pair $\left\langle L_{\infty}, L_{1}\right\rangle$ : paths are in $L_{\infty}$ and dual paths are in $L_{1}$. A change in this fundamental assumption would require a different approach. 
It follows from $(\mathbf{C . 2})-(\mathbf{C . 4})$ that

$$
(e, \delta e) \in C\left(s^{t}\right) \text { (a.s.) }
$$

where $\delta=M^{1-l} \gamma$ (because $\left|y_{l-1,1}\right| \leq M^{l-1}$ and $y_{l, 1} \geq \gamma e$ ), which yields $E_{t}\left|p_{t+1}\right| \leq$ $\delta^{-1}\left|p_{t}\right|$ (a.s.) for any sequence of measurable functions $p_{1}\left(s^{1}\right), p_{2}\left(s^{2}\right), \ldots$ satisfying (1.3). Consequently, for any such sequence we have $E\left|p_{t}\right|<\infty, t=1,2, \ldots$, if and only if $E\left|p_{1}\right|<\infty$. It follows from (C.3) that $\lambda\left(s^{t}\right) \leq M$ (a.s.) for any balanced path (1.5), and so the functional $E \ln \lambda\left(s^{t}\right)$ is well-defined (but, generally, may take on the value $-\infty)$.

The main result of this paper is the following theorem.

Theorem 1. Under assumptions (C.1) - (C.4), a von Neumann equilibrium exists.

Previous results of this kind (see [13] and references therein) were obtained under certain assumptions of strict convexity of the cones $C\left(s^{t}\right)$. Under these assumptions, an equilibrium not only exists, but is also in a sense stable. Conditions (C.1) (C.4), which are fully analogous to those used in the deterministic case (see, e.g., $[21,22,27])$, do no guarantee stability. The question of whether they are sufficient for the existence of equilibrium in the stochastic case was left open. Theorem 1 gives a positive answer to this question.

The strategy of the proof of Theorem 1 which we follow is classical and goes back to Dvoretzky, Wald and Wolfowitz [5]. It is based on the idea of "elimination of randomization". We first construct an appropriate extension, $\sigma_{t}=\left(s_{t}, s_{t}^{\prime}\right), t=$ $0, \pm 1, \ldots$, of the given stochastic process $\ldots, s_{0}, s_{1}, \ldots$ such that the new dynamical system defined in terms of $\ldots, \sigma_{0}, \sigma_{1}, \ldots$ possesses an equilibrium. The auxiliary process $\ldots, s_{0}^{\prime}, s_{1}^{\prime}, \ldots$ serves as an additional source of randomness. Then, using some subtle properties of convexity, we eliminate randomization and establish the existence of equilibrium in the original system.

The paper is organized as follows. In Section 2 we discuss links between the notions of a dual path and equilibrium introduced above and somewhat different but closely related notions considered in the literature - this is needed in order to make use of some results of previous work. In Section 3, we state the results of previous studies we employ and outline the plan of the proof of the main theorem. Section 4 contains some auxiliary propositions. In Section 5 we establish a key result needed for proving Theorem 1 . The proof is completed in Section 6 . Section 7 discusses the question of uniqueness of an equilibrium growth factor. The Appendix contains statements of some general facts used in this work.

\section{DYNKIN'S AND RADNER'S APPROACHES TO DUALITY}

In the definition of a dual path given in Section 1, we essentially follow the approach of Dynkin [6,7]. Another version of this concept was introduced by Radner (see, e.g., [25, 26]). Dynkin and Radner both dealt with somewhat different models, and when referring to their work, we adjust their considerations for the present context. According to Radner's approach (as applied in our context), a dual path is defined as a sequence of non-negative measurable vector functions $q_{0}\left(s^{0}\right), q_{1}\left(s^{1}\right), \ldots$ such that $E\left|q_{t}\left(s^{t}\right)\right|<\infty$ and

$$
E_{t-1}\left(q_{t} v\right) \leq q_{t-1} u \text { (a.s.) }
$$


for all $(u, v) \in Z_{t}$. We will refer to such sequences as $R$-dual paths. Since $C\left(s^{t}\right)$ is a cone, the set $Z_{t}$ contains with each pair of vectors $(u, v)$ all pairs $(\lambda u, \lambda v)$, where $\lambda\left(s^{t-1}\right) \geq 0$ is any bounded measurable scalar function. This implies that condition (2.1) is equivalent to

$$
E\left(q_{t} v\right) \leq E\left(q_{t-1} u\right),(u, v) \in Z_{t} .
$$

An $R$-dual path is balanced if there are measurable vector and scalar functions $q\left(s^{0}\right) \geq 0$ and $\lambda\left(s^{1}\right)>0$ such that

$$
q_{0}\left(s^{0}\right)=q\left(s^{0}\right), q_{t}\left(s^{t}\right)=\frac{q\left(s^{t}\right)}{\lambda\left(s^{t}\right) \ldots \lambda\left(s^{1}\right)}, t=1,2, \ldots,
$$

and $E\left|q\left(s^{0}\right)\right|<\infty$. These definitions lead to the following version of the concept of a von Neumann equilibrium. Let us say that a triplet of measurable functions $x\left(s^{0}\right) \geq 0, q\left(s^{0}\right) \geq 0, \lambda\left(s^{1}\right)>0$ forms an $R$-equilibrium if $E\left|q\left(s^{0}\right)\right|<\infty$, conditions (1.6) and (1.7) hold,

$$
q\left(s^{0}\right) x\left(s^{0}\right)=1 \text { (a.s.) }
$$

and

$$
E_{0} \frac{q\left(s^{1}\right) v\left(s^{1}\right)}{\lambda\left(s^{1}\right)} \leq q\left(s^{0}\right) u\left(s^{0}\right) \quad \text { (a.s.) }
$$

for all $\left(u\left(s^{0}\right), v\left(s^{1}\right)\right)$ in $Z_{1}$. By virtue of condition (2.5), the sequence (2.3) is an $R$-dual balanced path, and in view of (2.4) it supports the balanced path (1.5) in the sense that $q_{t} x_{t}=1$ (a.s.) for all $t \geq 0$.

Radner's approach appears to be more natural in the economic applications: $R$-dual paths admit a clear interpretation as price systems supporting optimal trajectories of economic dynamics. In this connection, most of the studies on stochastic von Neumann-Gale dynamical systems have dealt with what we call here $R$-dual paths and $R$-equilibria. However, it has been recently shown [4] that Dynkin's approach is preferable in the applications related to finance. In the present work, this approach serves as a powerful mathematical tool, playing a key role in the proof of the main existence theorem. Dealing with the notion of equilibrium defined in accordance with Dynkin's concept of duality, we employ a number of known results about $R$-equilibria and the following fact.

Theorem 2. An equilibrium exists if and only if an $R$-equilibrium exists.

Thus, the two notions of equilibrium are essentially equivalent. However, there is an asymmetry between the "if" and "only if" assertions in the above theorem. Given an equilibrium, one can immediately construct an $R$-equilibrium by taking some conditional expectations. To construct the former from the latter, one needs a more sophisticated argument using Lagrangian duality for convex variational problems in spaces of measurable functions (see Proposition 1 below).

Proof of Theorem 2. Let $x\left(s^{0}\right) \geq 0, p\left(s^{1}\right) \geq 0, \lambda\left(s^{1}\right)>0$ be a triplet of measurable functions forming an equilibrium, i.e. satisfying (1.6), (1.7), (1.9), (1.10), and (1.11). Define $q\left(s^{0}\right)=E\left(p\left(s^{1}\right) \mid s^{0}\right)$. By virtue of $(1.10),\left[E_{1} p\left(s^{2}\right)\right] v\left(s^{1}\right) / \lambda\left(s^{1}\right) \leq$ $p\left(s^{1}\right) u\left(s^{0}\right)$ (a.s.) for all $\left(u\left(s^{0}\right), v\left(s^{1}\right)\right)$ in $Z_{1}$. By taking the conditional expectation $E\left(\cdot \mid s^{0}\right)$ of both sides of the last inequality and using the fact that $E\left[p\left(s^{2}\right) \mid s^{1}\right]=$ $q\left(s^{1}\right)$, we obtain (2.5). Condition (2.4) holds as a consequence of (1.11), and so $x\left(s^{0}\right), q\left(s^{0}\right), \lambda\left(s^{1}\right)$ is an $R$-equilibrium. 
To prove the converse assertion, consider an $R$-equilibrium $x\left(s^{0}\right), q\left(s^{0}\right)$, $\lambda\left(s^{1}\right)$ and define $r\left(s^{1}\right):=q\left(s^{1}\right) / \lambda\left(s^{1}\right)$. We have $E\left|r\left(s^{1}\right)\right|<\infty$ by virtue of $(2.5)$, (1.13) and the fact that $E\left|q\left(s^{0}\right)\right|<\infty$. From (2.5) we obtain:

$$
E\left[r\left(s^{1}\right) v\left(s^{1}\right)-q\left(s^{0}\right) u\left(s^{0}\right)\right] \leq 0
$$

for all $\left(u\left(s^{0}\right), v\left(s^{1}\right)\right)$ in $Z_{1}$. By virtue of Proposition 1 we prove below, this implies the existence of a measurable vector function $g\left(s^{1}\right)$ such that $E\left|g\left(s^{1}\right)\right|<$ $\infty, E_{0} g\left(s^{1}\right)=0$ (a.s.) and

$$
E\left[r\left(s^{1}\right) v\left(s^{1}\right)-q\left(s^{0}\right) u\left(s^{1}\right)\right]-E g\left(s^{1}\right) u\left(s^{1}\right) \leq 0
$$

for all essentially bounded measurable $\left(u\left(s^{1}\right), v\left(s^{1}\right)\right)$ such that $\left(u\left(s^{1}\right), v\left(s^{1}\right)\right) \in$ $C\left(s^{1}\right)$ (a.s.). We will denote the class of such functions $\left(u\left(s^{1}\right), v\left(s^{1}\right)\right)$ by $W$.

Define $p\left(s^{1}\right)=q\left(s^{0}\right)+g\left(s^{1}\right)$. We claim that $x\left(s^{0}\right), p\left(s^{1}\right), \lambda\left(s^{1}\right)$ is an equilibrium. To prove this, from $(2.7)$ we obtain

$$
E \frac{q\left(s^{1}\right) v\left(s^{1}\right)}{\lambda\left(s^{1}\right)}-E p\left(s^{1}\right) u\left(s^{1}\right) \leq 0
$$

for all $(u, v) \in W$. Since $\left(u\left(s^{1}\right), 0\right) \in C\left(s^{1}\right)$ for any non-negative $u\left(s^{1}\right)$ (this follows from $(\mathbf{C . 1})$ and $(\mathbf{C . 2}))$, the last inequality implies $E p\left(s^{1}\right) u\left(s^{1}\right) \geq 0$ for any bounded measurable $u\left(s^{1}\right) \geq 0$. Consequently, $p\left(s^{1}\right) \geq 0$ (a.s.). Denote by $\hat{C}\left(s^{1}\right)$ the set of those $(a, b) \in C\left(s^{1}\right)$ which satisfy $|a| \leq 1$ and by $\hat{W}$ the class of all measurable functions $\left(u\left(s^{1}\right), v\left(s^{1}\right)\right)$ such that $\left(u\left(s^{1}\right), v\left(s^{1}\right)\right) \in \hat{C}\left(s^{1}\right)$ (a.s.). By virtue of (C.3) all such $(u, v)$ are essentially bounded, and so $\hat{W} \subseteq W$. Inequality (2.8), holding for all functions $(u, v) \in \hat{W}$, implies that for almost all $s^{1}$,

$$
\frac{q\left(s^{1}\right) b}{\lambda\left(s^{1}\right)}-p\left(s^{1}\right) a \leq 0 \text { for all }(a, b) \in C\left(s^{1}\right) \text { with }|a| \leq 1
$$

(see Proposition A.5 in the Appendix).

We can drop the constraint $|a| \leq 1$ in (2.9) because $C\left(s^{1}\right)$ is a cone. Since $E_{0} p\left(s^{1}\right)=E_{0}\left[q\left(s^{0}\right)+g\left(s^{1}\right)\right]=q\left(s^{0}\right)$ (a.s.), we have $E_{1} p\left(s^{2}\right)=q\left(s^{1}\right)$ (a.s.). Thus, with probability one,

$$
\frac{\left[E_{1} p\left(s^{2}\right)\right] b}{\lambda\left(s^{1}\right)}-p\left(s^{1}\right) a \leq 0 \text { for all }(a, b) \in C\left(s^{1}\right),
$$

which yields (1.10). By setting $a=x\left(s^{0}\right)$ and $b=\lambda\left(s^{1}\right) x\left(s^{1}\right)$ in (2.9), we get $1=$ $q\left(s^{1}\right) x\left(s^{1}\right) \leq p\left(s^{1}\right) x\left(s^{0}\right)$ a.s. (see (2.4)). On the other hand,

$$
E p\left(s^{1}\right) x\left(s^{0}\right)=E\left\{E_{0}\left[p\left(s^{1}\right)\right]\right\} x\left(s^{0}\right)=E q\left(s^{0}\right) x\left(s^{0}\right)=1 .
$$

Therefore $p\left(s^{1}\right) x\left(s^{0}\right)=1$ (a.s.), which proves (1.11). Thus $x\left(s^{0}\right), p\left(s^{1}\right), \lambda\left(s^{1}\right)$ is an equilibrium.

In the class $W$ of essentially bounded measurable vector functions $(u, v)$ satisfying $\left(u\left(s^{1}\right), v\left(s^{1}\right)\right) \in C\left(s^{1}\right)$ (a.s.), consider the subclass $W_{0}$ consisting of those $(u, v)$ for which there exists a measurable function $u^{\prime}\left(s^{0}\right)$ coinciding with $u\left(s^{1}\right)$ (a.s.). Clearly, those and only those $(u, v)$ in $W$ belong to $W_{0}$ which satisfy $u\left(s^{1}\right)=E_{0} u\left(s^{1}\right)$ (a.s.). From (2.6), we get

$$
F(u, v):=E\left[r\left(s^{1}\right) v\left(s^{1}\right)-q\left(s^{0}\right) u\left(s^{1}\right)\right] \leq 0
$$

for all $(u, v) \in W_{0}$. In the course of the proof of Theorem 2, we used the following fact. 
Proposition 1. If inequality (2.10) holds for all $(u, v) \in W_{0}$, then there exists an integrable vector function $g\left(s^{1}\right)$ such that $E_{0} g\left(s^{1}\right)=0$ (a.s.) and inequality (2.7) holds for all $(u, v) \in W$.

Inequality (2.10) says that the maximum of the functional $F(u, v)$ on the set of $(u, v) \in W$ satisfying the "non-anticipativity constraint" $u\left(s^{1}\right)=E_{0} u\left(s^{1}\right)$ (a.s.) is zero. The function $g\left(s^{1}\right)$ plays the role of a Lagrange multiplier relaxing this constraint. Lagrange multipliers of this kind were first considered by Rockafellar and Wets [28]. In the proof below, we use techniques outlined in [10].

Proof of Proposition 1. Denote by $L_{\infty}^{k}(t)$ the Banach space of essentially bounded measurable vector functions $x\left(s^{t}\right)$ with values in $\mathbb{R}^{k}$. We will regard functions $w=(u, v)$ in $W$ as elements of $L_{\infty}^{2 n}(1)$. Consider the operator $B$ transforming $w=(u, v) \in W$ into $B w:=u\left(s^{1}\right)-E_{0} u\left(s^{1}\right)$. This is a continuous linear operator of $L_{\infty}^{2 n}(1)$ into $L_{\infty}^{n}(1)$. We can characterize $W_{0}$ as the set of those $w \in W$ for which $B w=0$. According to (2.10), the function $\bar{w}:=0$ is a solution to the problem of maximization of the linear functional $F(z)$ on the convex set $W$ subject to the linear constraint $B w=0$. To analyze this problem we will use a version of the KuhnTucker theorem formulated in the Appendix as Proposition A.6. We will apply this result to the Banach spaces $D_{1}:=L_{\infty}^{2 n}(1)$ and $D_{2}:=L_{\infty}^{n}(1)$. Condition (a) of Proposition A.6 follows from the fact that the set $C\left(s^{1}\right)$ contains $\left\{(a, b) \in \mathbb{R}_{+}^{2 n}\right.$ : $a \geq e, 0 \leq b \leq \delta e\}$ (see (1.13)), which implies that the constant vector function $\tilde{w}:=(2 e, \delta e / 2)$ is an interior point of $W$ satisfying $B w=0$. Indeed, the open ball $\tilde{W}$ in $L_{\infty}^{2 n}(1)$ consisting of those $w$ for which ess $\sup \left|w\left(s^{1}\right)-\tilde{w}\right|<\delta / 2$ is contained in $W$ (we may assume without loss of generality that $\delta<1$ ). Assumption (b) is fulfilled because the image $B\left(D_{1}\right)$ is a closed subspace of $L_{\infty}^{n}(1)$. It consists of those $u \in L_{\infty}^{n}(1)$ for which $E_{0} u=0$. Requirement (c) is satisfied since $F$ is bounded on $\tilde{W}$ in view of integrability of $r\left(s^{1}\right)$ and $q\left(s^{0}\right)$ (see (2.10)). Thus, by virtue of Proposition A.6, there exists a functional $\pi \in\left[L_{\infty}^{2 n}(1)\right]^{*}$ such that $F(w)+\langle\pi, B w\rangle \leq F(\bar{w})$ for each $w \in W$.

Consider the decomposition $\pi=\pi^{a}+\pi^{s}$ of $\pi$ as described in Proposition A.7. Let $h\left(s^{1}\right)$ be an integrable function with values in $\mathbb{R}^{n}$ such that $\left\langle\pi^{a}, u\right\rangle=E h u$ for all $u \in L_{\infty}^{n}(1)$, and let $\chi^{k}\left(s^{1}\right)(k=1,2, \ldots)$ be measurable functions with values 0 and 1 satisfying

$$
\left\langle\pi^{s}, u\right\rangle=\left\langle\pi^{s}, \chi^{k} u\right\rangle, E \chi^{k} \rightarrow 0,\left\|\left(1-\chi^{k}\right) E_{0} \chi^{k}\right\|_{\infty} \rightarrow 0 .
$$

The functions $\chi^{k}$ are the indicators of the sets $\Gamma_{k}$ described in Proposition A.7 $\left(\mathcal{G}\right.$ being the $\sigma$-algebra generated by $\left.s^{0}\right)$. Fix any $w=(u, v) \in W$ and define $w^{\prime}:=\left(u^{\prime}, v^{\prime}\right):=\left(E_{0} u, 0\right)$,

$$
w_{k}:=\left(u_{k}, v_{k}\right)=\left(1-\chi^{k}\right) w+\chi^{k} w^{\prime}(k=1,2, \ldots) .
$$

We have $w^{\prime}, w_{k} \in W$, and so

$$
F\left(w_{k}\right)+\left\langle\pi^{a}, u_{k}-E_{0} u_{k}\right\rangle+\left\langle\pi^{s}, u_{k}-E_{0} u_{k}\right\rangle=F\left(w_{k}\right)+\left\langle\pi, B w_{k}\right\rangle \leq 0 .
$$

Since the functions $w_{k}$ are uniformly bounded and converge to $w$ in $L_{1}$, we have $F\left(w_{k}\right) \rightarrow F(w)$ (see (2.10)) and $\left\langle\pi^{a}, u_{k}-E_{0} u_{k}\right\rangle \rightarrow\left\langle\pi^{a}, u-E_{0} u\right\rangle$. Let us show that $\left\langle\pi^{s}, u_{k}-E_{0} u_{k}\right\rangle=\left\langle\pi^{s}, \chi^{k}\left(u_{k}-E_{0} u_{k}\right)\right\rangle \rightarrow 0$. Since $\pi^{s}$ is continuous in $\|\cdot\|_{\infty}$, it is sufficient to verify that $\left\|\psi^{k}\right\|_{\infty} \rightarrow 0$, where $\psi^{k}:=\chi^{k}\left(u_{k}-E_{0} u_{k}\right)$. We have $\psi^{k}=0$ 
when $\chi^{k}=0$. If $\chi^{k}=1$, then

$$
\begin{aligned}
\psi^{k} & \left.=E_{0} u-E_{0}\left(1-\chi^{k}\right) u-E_{0}\left(\chi^{k} E_{0} u\right)=E_{0}\left[u \chi^{k}-u E_{0} \chi^{k}\right)\right] \\
& =E_{0}\left(1-\chi^{k}\right) E_{0}\left(\chi^{k} u\right)-E_{0}\left[\left(1-\chi^{k}\right) u E_{0} \chi^{k}\right] .
\end{aligned}
$$

Consequently, $\left\|\psi^{k}\right\|_{\infty} \leq 2\|u\|_{\infty} \cdot\left\|\left(1-\chi^{k}\right) E_{0} \chi^{k}\right\|_{\infty} \rightarrow 0$. Thus, by passing to the limit in (2.11), we obtain

$$
F(u, v)+E h\left[u-E_{0} u\right] \leq 0 \text { for all }(u, v) \in W .
$$

Define $g\left(s^{1}\right):=E_{0} h\left(s^{1}\right)-h\left(s^{1}\right)$. Inequality (2.7) follows from (2.12) in view of the identity $E h\left[u-E_{0} u\right]=E\left[h-E_{0} h\right] u$.

\section{Plan of the proof of the MAin Result}

In the proof of Theorem 1, we will use several results of previous work, first of all, Theorem 3 below. Recall that throughout the paper we always assume that conditions (C.1) - (C.4) hold.

Theorem 3. If a von Neumann path exists, then an equilibrium exists.

The existence of an $R$-equilibrium in a system possessing a von Neumann path is proved in [2, Theorem 1]. Theorem 3 follows from this result and Theorem 2.

To formulate the next result let us introduce some definitions. Let $\mathcal{D}$ denote the stochastic von Neumann-Gale dynamical system defined by the stationary stochastic process $\ldots, s_{-1}, s_{0}, s_{1}, \ldots$ and the cone $C\left(s^{0}\right)$ which are supposed to be fixed throughout the paper. Suppose that together with the random sequence $. ., s_{-1}, s_{0}, s_{1}, \ldots$, we have another random sequence $. ., s_{-1}^{\prime}, s_{0}^{\prime}, s_{1}^{\prime}, \ldots$ with values in some measurable space. In other words, suppose we are given a probability measure $Q$ on the space of paths

$$
\sigma^{\infty}:=\left(\sigma_{t}\right)_{-\infty}^{+\infty}, \sigma_{t}:=\left(s_{t}, s_{t}^{\prime}\right),
$$

of the process $\ldots, \sigma_{-1}, \sigma_{0}, \sigma_{1}, \ldots$ such that the projection of $Q$ on the space of paths $s^{\infty}:=\left(s_{t}\right)_{-\infty}^{+\infty}$ of the original process $\ldots, s_{-1}, s_{0}, s_{1}, \ldots$ coincides with the given measure $P$. We shall say that the process..., $\sigma_{-1}, \sigma_{0}, \sigma_{1}, \ldots$ is non-anticipative (with respect to $\left.\ldots, s_{-1}, s_{0}, s_{1}, \ldots\right)$ if, for each bounded measurable function $g\left(\sigma^{t}\right)$, we have

$$
E\left[g\left(\sigma^{t}\right) \mid s^{\infty}\right]=E\left[g\left(\sigma^{t}\right) \mid s^{t}\right], t=0, \pm 1, \ldots \text { (a.s.) },
$$

where $\sigma^{t}=\left(\ldots, \sigma_{t-1}, \sigma_{t}\right)$ and here $E(\cdot \mid \cdot)$ stands for the conditional expectation with respect to the measure $Q$. Relation (3.2) almost surely holds with respect to $Q$, or equivalently with respect to $P$, because the projection of $Q$ on the space of paths $s^{\infty}$ coincides with $P$. Equality (3.2) means that if we wish to predict $\sigma^{t}$ based on information about $\ldots, s_{-1}, s_{0}, s_{1}, \ldots$, then what matters is only $s^{t}=\left(\ldots, s_{t-1}, s_{t}\right)$ the past and the present of the given process $\left(s_{t}\right)$, the probabilistic forecast of $\sigma^{t}$ being independent of the future $s_{t+1}, s_{t+2}, \ldots$ of it.

Given a stationary non-anticipative process $\sigma_{t}=\left(s_{t}, s_{t}^{\prime}\right), t=0, \pm 1, \pm 2, \ldots$, define

$$
\bar{C}\left(\sigma^{t}\right)=C\left(s^{t}\right) .
$$

Consider the system $\overline{\mathcal{D}}$ specified in terms of the stochastic process $\sigma_{t}=\left(s_{t}, s_{t}^{\prime}\right)$, $t=0, \pm 1, \pm 2, \ldots$, and the random cone (3.3). This dynamical system will be called the extension of $\mathcal{D}$ corresponding to the stationary non-anticipative process $\left(\sigma_{t}\right)$. Note that, in the extended system, the random cone $\bar{C}\left(\sigma^{t}\right)=C\left(s^{t}\right)$ does not depend 
on the process $\left(s_{t}^{\prime}\right)$. However, the class of paths in $\overline{\mathcal{D}}$ is of course larger than in $\mathcal{D}$ : these paths might depend not only on $\left(s_{t}\right)$, but also on $\left(s_{t}^{\prime}\right)$.

Theorem 4. There exists a stationary non-anticipative extension $\sigma_{t}=\left(s_{t}, s_{t}^{\prime}\right), t=$ $0, \pm 1, \ldots$ of the process $s_{t}, t=0, \pm 1, \ldots$, such that the extended dynamical system $\overline{\mathcal{D}}$ defined in terms of $\left(\sigma_{t}\right)$ has a von Neumann path and hence an equilibrium.

The analogous result pertaining to $R$-equilibria is established in [12, Theorem 2 ]. Combined with Theorem 3, it implies the existence of an extension $\overline{\mathcal{D}}$ of the original system $\mathcal{D}$ possessing an equilibrium.

The plan of the proof of Theorem 1 is as follows. Consider an extension $\overline{\mathcal{D}}$ of the given system $\mathcal{D}$ (defined in terms of an appropriate non-anticipative process $\left.\left(\sigma_{t}\right)\right)$ in which an equilibrium $x\left(\sigma^{0}\right), p\left(\sigma^{1}\right), \lambda\left(\sigma^{1}\right)$ exists. Let $x_{0}, x_{1}, \ldots$ be the von Neumann path in $\overline{\mathcal{D}}$ defined by $x_{0}\left(\sigma^{0}\right)=x\left(\sigma^{0}\right)$ and $x_{t}\left(\sigma^{t}\right)=\lambda\left(\sigma^{t}\right) \ldots \lambda\left(\sigma^{1}\right) x\left(\sigma^{t}\right)$. Put $w_{t}\left(s^{t}\right)=E\left(x_{t}\left(\sigma^{t}\right) \mid s^{t}\right)(t=0,1, \ldots)$. We show (Proposition 5) that $w_{0}, w_{1}, \ldots$ is a path in $\overline{\mathcal{D}}$ and $\mathcal{D}$, and there exists a constant $d \geq 0$ such that $E \ln \left|w_{t}\left(s^{t}\right)\right|-$ $t E \ln \lambda\left(\sigma^{t}\right) \geq-d$ for all $t$. The last inequality means that the path $w_{0}, w_{1}, \ldots$ cannot grow "infinitely slower" than $x_{0}, x_{1}, \ldots$. We consider the $L_{1}$ closure $\operatorname{cl} \mathcal{L}$ of the convex hull $\mathcal{L}$ of the set functions $\ln \left|w_{t}\left(s^{1}\right)\right|-\ln \left|w_{t-1}\left(s^{0}\right)\right|$. By using the above growth property of $w_{0}, w_{1}, \ldots$, we prove (Theorems 5 and 6 ) that $\lambda\left(\sigma^{1}\right)$ is homological to some function $\mu\left(s^{1}\right)$ in $\operatorname{cl} \mathcal{L}$. Two functions $\lambda\left(\sigma^{1}\right)$ and $\lambda^{\prime}\left(\sigma^{1}\right)$ are said to be homological if $\lambda^{\prime}\left(\sigma^{1}\right)=\phi\left(\sigma^{0}\right) \lambda\left(\sigma^{1}\right) \phi\left(\sigma^{1}\right)^{-1}$ (a.s.) for some measurable $\phi\left(\sigma^{0}\right)>0$. Since all elements in $\mathcal{L}$ are measurable functions of $s^{1}$, every element in the $L_{1}$ closure of $\mathcal{L}$ can be represented by a measurable function of $s^{1}$. Having proved that $\lambda\left(\sigma^{1}\right)$ is homological to a measurable function of $s^{1}$, we derive from this (Theorem 7) that there exists a pair $\check{x}\left(s^{0}\right), \check{\lambda}\left(s^{1}\right)$ generating a balanced path in $\mathcal{D}$ with $E \ln \check{\lambda}\left(s^{1}\right)=E \ln \lambda\left(\sigma^{1}\right)$. Since $\lambda\left(\sigma^{1}\right)$ maximizes $E \ln \lambda\left(\sigma^{1}\right)$ in $\overline{\mathcal{D}}$, the last equality shows that $\check{x}\left(s^{0}\right), \check{\lambda}\left(s^{1}\right)$ generates a von Neumann path in $\mathcal{D}$. By virtue of Theorem 3 , this implies the existence of an equilibrium in $\mathcal{D}$.

\section{SOME AUXILIARY PROPOSITIONS}

Proposition 2. Let $(x, p, \lambda)$ be an equilibrium. Then there exists a constant $\kappa>0$ such that $p\left(s^{1}\right) \geq \kappa e$ and $\lambda\left(s^{1}\right) \geq \kappa$ (a.s).

Proof. The equalities $p\left(s^{1}\right) x\left(s^{0}\right)=1$ and $\left|x\left(s^{0}\right)\right|=1$ imply

$$
\left|p\left(s^{1}\right)\right|=\left|p\left(s^{1}\right)\right|\left|x\left(s^{0}\right)\right| \geq p\left(s^{1}\right) x\left(s^{0}\right)=1 .
$$

Here and in what follows we often omit "a.s." when this does not lead to ambiguity. Consider the functions $y_{1, i}, \ldots, y_{l, i}$ described in (C.4) and the sequence $p_{1}, p_{2}, \ldots$ defined by (1.8). Since $p_{1} e_{i}, p_{2} y_{1, i}, \ldots, p_{l+1} y_{l, i}$ is a supermartingale, we have

$$
E\left(\frac{p\left(s^{l+1}\right) y_{l, i}}{\lambda\left(s^{l}\right) \ldots \lambda\left(s^{1}\right)} \mid s^{1}\right)=E\left(p_{l+1} y_{l, i} \mid s^{1}\right) \leq p_{1} e_{i}=p^{i}\left(s^{1}\right),
$$

where $p^{i}\left(s^{1}\right)$ is the $i$ th coordinate of $p\left(s^{1}\right)$. In the above formula, $p\left(s^{l+1}\right) y_{l, i} \geq$ $\gamma\left|p\left(s^{l+1}\right)\right| \geq \gamma$ and $\lambda\left(s^{l}\right) \ldots \lambda\left(s^{1}\right) \leq M^{l}$. Consequently, $p^{i}\left(s^{1}\right) \geq \alpha$, where $\alpha=$ $\gamma M^{-l}, i=1, \ldots, n$.

To show that $\lambda\left(s^{1}\right)$ is uniformly bounded away from zero define

$$
y_{t}\left(s^{t}\right)=\sum_{i=1}^{n} x^{i}\left(s^{0}\right) y_{t, i}\left(s^{t}\right), t=1, \ldots, l .
$$


By virtue of convexity of $C\left(s^{t}\right)$ and (1.6), we have $\left(x_{0}, y_{1}\right) \in Z_{1},\left(y_{1}, y_{2}\right) \in Z_{2}, \ldots$, $\left(y_{l-1}, y_{l}\right) \in Z_{l}, y_{l} \geq \gamma e$, where $x_{0}=x\left(s^{0}\right)$. In view of $(\mathbf{C . 3}),|\gamma e| \leq M^{l-1}\left|y_{1}\left(s^{1}\right)\right|$ and so $\left|y_{1}\left(s^{1}\right)\right| \geq M^{1-l} \gamma$. From (1.10) and (1.11), we get

$$
\lambda\left(s^{1}\right)^{-1}\left[E_{1} p\left(s^{2}\right)\right] y_{1}\left(s^{1}\right) \leq p\left(s^{1}\right) x\left(s^{0}\right)=1,
$$

which implies $\lambda\left(s^{1}\right) \geq\left[E_{1} p\left(s^{2}\right)\right] y_{1}\left(s^{1}\right) \geq \alpha\left|y_{1}\left(s^{1}\right)\right| \geq \alpha M^{1-l} \gamma$. It remains to define $\kappa$ as the smallest of the numbers $\alpha M^{1-l} \gamma$ and $\alpha$.

Proposition 3. If $(x, p, \lambda)$ is an equilibrium, then the sequence defined by (1.5) is a von Neumann path.

Proof. Consider a pair of measurable functions $x^{\prime}\left(s^{0}\right) \geq 0, \lambda^{\prime}\left(s^{1}\right)>0$ generating some balanced path. Then $\left|x^{\prime}\left(s^{0}\right)\right|=1$ (a.s.) and $\left(x^{\prime}\left(s^{0}\right), \lambda^{\prime}\left(s^{1}\right) x^{\prime}\left(s^{1}\right)\right) \in$ $C\left(s^{1}\right)$ (a.s.). By using (1.10), we find

$$
\lambda^{\prime}\left(s^{1}\right) \lambda\left(s^{1}\right)^{-1} E\left(p\left(s^{2}\right) \mid s^{1}\right) x^{\prime}\left(s^{1}\right) \leq p\left(s^{1}\right) x^{\prime}\left(s^{0}\right),
$$

which yields

$$
E \ln \left[\lambda^{\prime}\left(s^{1}\right) \lambda\left(s^{1}\right)^{-1}\right] \leq E \ln p\left(s^{1}\right) x^{\prime}\left(s^{0}\right)-E \ln \left[E\left(p\left(s^{2}\right) x^{\prime}\left(s^{1}\right) \mid s^{1}\right)\right],
$$

where $E \ln p\left(s^{1}\right) x^{\prime}\left(s^{0}\right)=E \ln p\left(s^{2}\right) x^{\prime}\left(s^{1}\right)=E\left[E \ln \left(p\left(s^{2}\right) x^{\prime}\left(s^{1}\right) \mid s^{1}\right)\right]$. By applying to $\xi:=p\left(s^{2}\right) x^{\prime}\left(s^{1}\right)$ and $f(x):=\ln x$ Jensen's inequality (see Proposition A.2, part (a), in the Appendix), we obtain that the right-hand side of (4.2) is not greater than zero. (Here, $\kappa \leq \xi \leq\left|p\left(s^{2}\right)\right|$, and so $E \xi<\infty$ and $E|\ln \xi|<\infty$.)

Let $(x, p, \lambda)$ be a von Neumann equilibrium. A path $w_{0}, w_{1}, \ldots$ is called good (cf. Gale [15]) if

$$
E \ln \left|w_{t}\right|-t E \ln \lambda\left(s^{t}\right) \geq-d, t=0,1, \ldots,
$$

for some constant $d \geq 0$. According to (4.3), the von Neumann path defined by (1.5) does not grow "infinitely faster" than $w_{0}, w_{1}, \ldots$ (which motivates the above term).

We will denote by $T$ the operator transforming a function $x\left(s^{t}\right)$ into $x\left(s^{t+1}\right)$ $(t=0, \pm 1, \ldots)$. If a measurable function $x$ is in $L_{1}$ (i.e. $\left.E|x|<\infty\right)$, then for any $m=0, \pm 1, \ldots$ the function $T^{m} x$ is in $L_{1}$ and

$$
E x=E\left(T^{m} x\right), T^{m} E\left(x\left(s^{t}\right) \mid s^{t-1}\right)=E\left(x\left(s^{t+m}\right) \mid s^{t+m-1}\right)
$$

by virtue of the stationarity of the process $\left(s_{t}\right)$.

Consider a good path $\left(w_{t}\right)$. It follows from (4.3) that $\left|w_{t}\right|>0$ (a.s.). For each $t=1,2, \ldots$, define

$$
\mu_{t}\left(s^{t}\right)=\frac{\left|w_{t}\left(s^{t}\right)\right|}{\left|w_{t-1}\left(s^{t-1}\right)\right|}, \mu^{t}\left(s^{1}\right)=T^{-(t-1)} \mu_{t}\left(s^{t}\right) .
$$

Proposition 4. The following inequalities are valid: $\mu^{t} \leq M$ (a.s.), $E\left|\ln \mu^{t}\right|<\infty$ and

$$
\left[\sum_{t=1}^{N} E \ln \mu^{t}\left(s^{1}\right)\right]-N E \ln \lambda\left(s^{1}\right) \geq-D, N=1,2, \ldots,
$$

for some constant $D \geq 0$. 
Proof. The first inequality follows from (C.3). To prove the second and the third, it is sufficient to prove the analogous inequalities for the random variables $\mu_{t}$ (see (4.4)). We have $\ln \mu_{t}=\ln \left|w_{t}\right|-\ln \left|w_{t-1}\right|$, where $\ln \left|w_{t}\right| \leq t \ln M+\ln \left|w_{0}\right|$ by virtue of (C.3). From (4.3) it follows that $E \ln \left|w_{t}\right|>-\infty$ and $E \ln \left|w_{t-1}\right|>-\infty$, which implies that $\ln \left|w_{t}\right|$ and $\ln \left|w_{t-1}\right|$ are in $L_{1}$, and so $\ln \mu_{t}$ is in $L_{1}$. Further, we have

$$
\begin{gathered}
\sum_{t=1}^{N} E \ln \mu_{t}=\sum_{t=1}^{N}\left[E \ln \left|w_{t}\right|-E \ln \left|w_{t-1}\right|\right] \\
=E \ln \left|w_{N}\right|-E \ln \left|w_{0}\right| \geq N E \ln \lambda-d-E \ln \left|w_{0}\right|,
\end{gathered}
$$

which shows that $D$ can be defined as $d+E|\ln | w_{0}||$.

\section{An approximation theorem}

Consider a von Neumann equilibrium $(x, p, \lambda)$, a good path $w_{0}, w_{1}, \ldots$ and the functions $\mu^{t}\left(s^{1}\right)$ defined by (4.5). Denote by $\mathcal{L}$ the convex hull of the set of functions $\left\{\ln \mu^{t}\left(s^{1}\right), t=1,2, \ldots\right\}$ and put $K\left(s^{1}\right)=\left|p\left(s^{1}\right)\right|$.

Theorem 5. There exists a sequence of measurable functions $\xi^{m}\left(s^{1}\right)$ in $\mathcal{L}(m=1,2, \ldots)$ and a measurable function $\phi\left(s^{0}\right)>0$ such that

$$
\xi^{m}\left(s^{1}\right) \rightarrow \ln \lambda\left(s^{1}\right)+\ln \phi\left(s^{0}\right)-\ln \phi\left(s^{1}\right)\left(L_{1}\right)
$$

and

$$
\kappa \leq \phi\left(s^{0}\right) \leq K\left(s^{1}\right) \text { (a.s.). }
$$

This result will be applied in Section 6 to an extension $\overline{\mathcal{D}}$ of the original dynamical system $\mathcal{D}$ in which an equilibrium exists. The essence of Theorem 5 lies in the following. According to this theorem, there exists a function, $\xi\left(s^{1}\right):=\ln \lambda\left(s^{1}\right)+$ $\ln \phi\left(s^{0}\right)-\ln \phi\left(s^{1}\right)$, having the following properties: (a) $\exp \left(\xi\left(s^{1}\right)\right)$ is homological to the von Neumann growth factor $\lambda\left(s^{1}\right)$, and (b) $\xi\left(s^{1}\right)$ can be approximated in $L_{1}$ by convex combinations $\xi^{m}\left(s^{1}\right)$ of the functions $\ln \mu^{t}\left(s^{1}\right), t=1,2, \ldots$, where $\mu^{t}\left(s^{1}\right)$ are the growth factors associated with a good path.

Proof of Theorem 5. Since $\left(w_{t-1}, w_{t}\right) \in Z_{t}$, we have

$$
\lambda\left(s^{t}\right)^{-1} E_{t} p\left(s^{t+1}\right) w_{t} \leq p\left(s^{t}\right) w_{t-1} .
$$

This inequality implies

$$
\ln \frac{\mu_{t}}{\lambda\left(s^{t}\right)}=\ln \frac{\left|w_{t}\right|}{\lambda\left(s^{t}\right)\left|w_{t-1}\right|} \leq \ln \frac{p\left(s^{t}\right) v_{t-1}}{E_{t} p\left(s^{t+1}\right) v_{t}}=-\ln \frac{E_{t} p\left(s^{t+1}\right) v_{t}}{p\left(s^{t}\right) v_{t-1}},
$$

where $v_{t}\left(s^{t}\right):=w_{t}\left|w_{t}\right|^{-1}$ and $\mu_{t}\left(s^{t}\right)=\left|w_{t}\right| /\left|w_{t-1}\right|$. Consequently, we have

$$
T^{-(t-1)} \ln \frac{\mu_{t}}{\lambda\left(s^{t}\right)} \leq T^{-(t-1)}\left[-\ln \frac{E_{t} p\left(s^{t+1}\right) v_{t}}{p\left(s^{t}\right) v_{t-1}}\right],
$$

which yields

$$
\begin{gathered}
\ln \frac{\mu^{t}}{\lambda\left(s^{1}\right)} \leq-\ln \frac{\left[E_{1} p\left(s^{2}\right)\right] T T^{-t} v_{t}}{p\left(s^{1}\right) T^{-(t-1)} v_{t-1}}=-\ln \frac{\left[E_{1} p\left(s^{2}\right)\right] T v^{t}}{p\left(s^{1}\right) v^{t-1}} \\
=-\ln \frac{E_{1}\left[p\left(s^{2}\right) T v^{t}\right]}{p\left(s^{1}\right) v^{t-1}}=-\ln \frac{E_{1}\left\{T\left[p\left(s^{1}\right) v^{t}\right]\right\}}{p\left(s^{1}\right) v^{t-1}}=-\ln \frac{E_{1}\left\{T \gamma^{t}\right\}}{\gamma^{t-1}},
\end{gathered}
$$


where $v^{t}\left(s^{0}\right):=T^{-t} v_{t}\left(s^{t}\right)$ and $\gamma^{t}\left(s^{1}\right):=p\left(s^{1}\right) v^{t}\left(s^{0}\right)$. Since $\left|v^{t}\left(s^{0}\right)\right|=1$, we have

$$
\kappa \leq \gamma^{t}\left(s^{1}\right) \leq K\left(s^{1}\right)\left[=\left|p\left(s^{1}\right)\right|\right],
$$

where $\kappa>0$ and $E K\left(s^{1}\right)<\infty$.

From (5.3) we obtain that the random variables $\nu^{t}\left(s^{1}\right):=\ln \mu^{t}\left(s^{1}\right)-\ln \lambda\left(s^{1}\right)$ satisfy

$$
\nu^{t} \leq-\ln \frac{E_{1}\left\{T \gamma^{t}\right\}}{\gamma^{t-1}}, t=1,2, \ldots
$$

and from (4.6) we get

$$
\sum_{t=1}^{N} E \nu^{t} \geq-D, N=1,2, \ldots
$$

To complete the proof of the theorem, it is sufficient to prove the following proposition.

Proposition 5. Let $\nu^{t}\left(s^{1}\right), t=1,2, \ldots$, be a sequence of functions in $L_{1}$ satisfying inequalities (5.5) and (5.6), where $D \geq 0$ is a constant and $\gamma^{t}\left(s^{1}\right), t=1,2, \ldots$, are measurable functions meeting requirement (5.4). Then there exists a measurable function $\phi\left(s^{0}\right)>0$ satisfying (5.2) and a sequence of measurable functions $\zeta^{m}\left(s^{1}\right)$ belonging to the convex hull of the set $\left\{\nu^{t}\left(s^{1}\right), t=0,1, \ldots\right\}$ such that $\zeta^{m}\left(s^{1}\right) \rightarrow$ $\ln \phi\left(s^{0}\right)-\ln \phi\left(s^{1}\right)$ in $L_{1}$.

Proof. From (5.5) we obtain

$$
\begin{gathered}
\sum_{t=1}^{N} \nu^{t} \leq-\sum_{t=1}^{N} \ln \frac{E_{1}\left(T \gamma^{t}\right)}{\gamma^{t-1}} \\
=-\sum_{t=1}^{N} \ln \frac{E_{1}\left(T \gamma^{t}\right)}{\gamma^{t}}-\sum_{t=1}^{N} \ln \frac{\gamma^{t}}{\gamma^{t-1}}=-\sum_{t=1}^{N} \ln \frac{E_{1}\left(T \gamma^{t}\right)}{\gamma^{t}}-\ln \gamma^{N}+\ln \gamma^{0},
\end{gathered}
$$

and so

$$
\xi_{N}:=\frac{1}{N} \sum_{t=1}^{N} \nu^{t} \leq-\frac{1}{N} \sum_{t=1}^{N}\left\{\ln \left[E_{1} e^{T \beta^{t}}\right]-\beta^{t}\right\}+\varepsilon_{N},
$$

where $\varepsilon_{N}:=\left(\ln \gamma^{0}-\ln \gamma^{N}\right) / N$ and $\beta^{t}\left(s^{1}\right):=\ln \gamma^{t}\left(s^{1}\right)$. The random variables $\varepsilon_{N}$ converge to zero a.s. and in $L_{1}$ because $\ln \kappa \leq \ln \gamma^{N} \leq \ln K\left(s^{1}\right)$ for all $N$ (see $(5.4))$.

By using the concavity of the operator $\xi \mapsto\left[-\ln \left(E_{1} e^{\xi}\right)\right]$ (see Proposition A.3 in the Appendix), we get

$$
-\frac{1}{N} \sum_{t=1}^{N} \ln \left[E_{1} e^{T \beta^{t}}\right] \leq-\ln \left[E_{1} e^{T \psi^{N}}\right] \text { (a.s.) }
$$

where $\psi^{N}\left(s^{1}\right):=N^{-1} \sum_{t=1}^{N} \beta^{t}\left(s^{1}\right)$. This inequality, combined with (5.7), yields

$$
\xi_{N} \leq-\ln \left(E_{1} e^{T \psi^{N}}\right)+\psi^{N}+\varepsilon_{N} .
$$

Note that the random variables $\gamma^{t}, \beta^{t}, T \beta^{t}, e^{T \beta^{t}}$ and $\psi^{N}$ are in $L_{1}$ because

$$
\kappa \leq \gamma^{t} \leq K\left(s^{1}\right), \ln \kappa \leq \beta^{t} \leq \ln K\left(s^{1}\right), \ln \kappa \leq \psi^{N} \leq \ln K\left(s^{1}\right) .
$$


It follows from (5.9) that $\left|\psi^{N}\left(s^{1}\right)\right|$ is bounded by an integrable random variable $\Psi\left(s^{1}\right):=|\ln \kappa|+\left|\ln K\left(s^{1}\right)\right|$.

By using the Komlós theorem (see Proposition A.1), we can select a sequence $N_{1}<N_{2}<\ldots$ such that

$$
\pi^{m}:=\frac{1}{m} \sum_{i=1}^{m} \psi^{N_{i}} \rightarrow \psi(\text { a.s. })
$$

for some $\psi=\psi\left(s^{1}\right)$ with $\ln \kappa \leq \psi\left(s^{1}\right) \leq \ln K\left(s^{1}\right)$. Since $\left|\psi^{N}\left(s^{1}\right)\right| \leq \Psi\left(s^{1}\right)$, where $E \Psi<\infty$, this convergence will also be in $L_{1}$. From (5.8), we get

$$
\begin{gathered}
\zeta^{m}\left(s^{1}\right):=\frac{1}{m} \sum_{i=1}^{m} \xi_{N_{i}} \leq \frac{1}{m} \sum_{i=1}^{m}\left[-\ln \left(E_{1} e^{T \psi^{N_{i}}}\right)\right]+\pi^{m}+\frac{1}{m} \sum_{i=1}^{m} \varepsilon_{N_{i}} \\
\leq-\ln \left(E_{1} e^{\frac{1}{m} \sum_{i=1}^{m} T \psi^{N_{i}}}\right)+\pi^{m}+\varepsilon^{m}=-\ln \left(E_{1} e^{T \pi^{m}}\right)+\pi^{m}+\varepsilon^{m},
\end{gathered}
$$

where $\varepsilon^{m}:=m^{-1} \sum_{i=1}^{m} \varepsilon_{N_{i}} \rightarrow 0$ a.s. and in $L_{1}$. The second inequality in the above chain of relations follows from the concavity of the operator $\xi \mapsto\left[-\ln \left(E_{1} e^{\xi}\right)\right]$ and the fact that $e^{\psi^{N}} \in L_{1}$ (see (5.9)). By virtue of (5.6), we have $E \xi_{N} \geq-D / N$, and so $E \zeta^{m} \geq-d_{m}$, where $d_{m}:=D m^{-1}\left(N_{1}^{-1}+\ldots+N_{m}^{-1}\right) \rightarrow 0$.

Put $\eta^{m}\left(s^{1}\right):=-\ln \left(E_{1} e^{T \pi^{m}}\right)+\pi^{m}+\varepsilon^{m}$ and $\theta\left(s^{1}\right):=e^{\psi\left(s^{1}\right)}$. Observe that $\kappa \leq \theta\left(s^{1}\right) \leq K\left(s^{1}\right)$ and

$$
\eta^{m} \rightarrow-\ln \left(E_{1} e^{T \psi}\right)+\psi=-\ln E_{1}(T \theta)+\ln \theta \text { a.s. and in } L_{1} .
$$

Indeed, $\pi^{m} \rightarrow \psi, \varepsilon^{m} \rightarrow 0$ a.s. and in $L_{1}$. The random variables $e^{T \pi^{m}} \geq 0$ converge to $e^{T \psi}$ a.s. and are bounded by the integrable random variable $K\left(s^{2}\right)$, consequently, $E_{1} e^{T \pi^{m}} \rightarrow E_{1} e^{T \psi}$ a.s. and in $L_{1}$. This implies $\ln E_{1} e^{T \pi^{m}} \rightarrow \ln E_{1} e^{T \psi}$ a.s. and in $L_{1}$ because $\kappa \leq E_{1} e^{T \pi^{m}} \leq E_{1} K\left(s^{2}\right)$ and $\ln \kappa \leq \ln E_{1} e^{T \pi^{m}} \leq \ln E_{1} K\left(s^{2}\right)$ (see (5.9) and (5.10)).

We have shown that $E \zeta^{m} \geq-d_{m}$, where $d_{m} \rightarrow 0$. Therefore and by virtue of (5.12),

$$
E\left(\eta^{m}-\zeta^{m}\right) \leq E \eta^{m}+d_{m} \rightarrow-E \ln E_{1}(T \theta)+E \ln \theta .
$$

In (5.11) we established that $\eta^{m}-\zeta^{m} \geq 0$. Consequently, $E \eta^{m}+d_{m} \geq 0$, and so $-E \ln E_{1}(T \theta)+E \ln \theta \geq 0$. On the other hand, Jensen's inequality (see Proposition A.2, assertion (a)) implies $\ln E_{1}(T \theta) \geq E_{1} \ln (T \theta)$ (a.s.). Thus

$$
E \ln E_{1}(T \theta) \geq E\left[E_{1} \ln (T \theta)\right]=E \ln (T \theta)=E \ln \theta,
$$

and so

$$
-E \ln E_{1}(T \theta)+E \ln \theta=0 .
$$

From (5.13) and (5.14), we find $0 \leq E\left(\eta^{m}-\zeta^{m}\right) \leq E \eta^{m}+d_{m} \rightarrow 0$, and so $E\left(\eta^{m}-\zeta^{m}\right) \rightarrow 0$, which means that $\eta^{m}-\zeta^{m} \rightarrow 0\left(L_{1}\right)$ because $\eta^{m}-\zeta^{m} \geq 0$.

We have shown that $\eta^{m}-\zeta^{m} \rightarrow 0$ in $L_{1}$. On the other hand, $\eta^{m} \rightarrow-\ln E_{1}(T \theta)+$ $\ln \theta$ in $L_{1}$ (see (5.12)). Consequently, $\zeta^{m} \rightarrow-\ln E_{1}(T \theta)+\ln \theta$ in $L_{1}$. The functions $\zeta^{m}\left(s^{1}\right)$ belong to the convex hull of the set $\left\{\nu^{t}\left(s^{1}\right), t=0,1, \ldots\right\}$ because

$$
\zeta^{m}=\frac{1}{m} \sum_{i=1}^{m} \frac{1}{N_{i}} \sum_{t=1}^{N_{i}} \nu^{t}
$$

(see (5.7) and (5.11)). 
It remains to show that there exists a measurable function $\phi\left(s^{0}\right)>0$ for which $\theta\left(s^{1}\right)=\phi\left(s^{0}\right)$ (a.s.). To this end observe that $E \ln E_{0} \theta=E \ln E_{1}(T \theta)=E \ln \theta=$ $E E_{0} \ln \theta$ by virtue of (5.14). On the other hand, Jensen's inequality (see Proposition A.2, part (a)) gives $\ln E_{0} \theta-E_{0} \ln \theta \geq 0$. Both random variables $\ln E_{0} \theta$ and $E_{0} \ln \theta$ are in $L_{1}$ because $\kappa \leq \theta\left(s^{1}\right) \leq K\left(s^{1}\right)$. Consequently, $\ln E_{0} \theta=E_{0} \ln \theta$, which in view of part (b) of Proposition A.2 implies the equality $E_{0} \theta=\theta$ (a.s.). Thus we can define $\phi\left(s^{0}\right)$ as $E_{0} \theta$. Inequality (5.2) follows from the analogous inequality for $\theta$.

\section{Existence of a VON NEUMANN PATH}

By virtue of Theorem 4, there exists a stationary non-anticipative extension $\sigma_{t}=$ $\left(s_{t}, s_{t}^{\prime}\right), t=0, \pm 1, \ldots$, of the process $\left(s_{t}\right)$, such that the extended dynamical system $\overline{\mathcal{D}}$ defined in terms of the process $\left(\sigma_{t}\right)$ possesses an equilibrium $x\left(\sigma^{0}\right), p\left(\sigma^{1}\right), \lambda\left(\sigma^{1}\right)$. Consider the von Neumann path $x_{0}, x_{1}, \ldots$ in $\overline{\mathcal{D}}$ defined by $x_{0}\left(\sigma^{0}\right)=x\left(\sigma^{0}\right)$ and $x_{t}\left(\sigma^{t}\right)=\lambda\left(\sigma^{t}\right) \ldots \lambda\left(\sigma^{1}\right) x\left(\sigma^{t}\right)$. Put

$$
w_{t}\left(s^{t}\right)=E\left(x_{t}\left(\sigma^{t}\right) \mid s^{t}\right), t=0,1, \ldots
$$

Proposition 6. The sequence $w_{0}, w_{1}, \ldots$ is a good path in the dynamical system $\overline{\mathcal{D}}$.

Proof. First of all, we observe that $w_{0}, w_{1}, \ldots$ is a path in $\overline{\mathcal{D}}$. Indeed, we have $\left(x_{t-1}\left(\sigma^{t-1}\right), x_{t}\left(\sigma^{t}\right)\right) \in C\left(s^{t}\right)$ (a.s.), and so $E\left[\left(x_{t-1}\left(\sigma^{t-1}\right), x_{t}\left(\sigma^{t}\right)\right) \mid s^{\infty}\right] \in C\left(s^{t}\right)$ (a.s.); see Proposition A.4 in the Appendix. Since the process $\left(\sigma_{t}\right)$ is non-anticipative with respect to $\left(s_{t}\right)$, we get $E\left[x_{t-1}\left(\sigma^{t-1}\right) \mid s^{\infty}\right]=E\left[x_{t-1}\left(\sigma^{t-1}\right) \mid s^{t-1}\right]=w_{t-1}$ and $E\left[x_{t}\left(\sigma^{t}\right) \mid s^{\infty}\right]=E\left[x_{t}\left(\sigma^{t}\right) \mid s^{t}\right]=w_{t}$. Thus we obtain $\left(w_{t-1}, w_{t}\right) \in C\left(s^{t}\right)$ (a.s.), which means that $\left(w_{t}\right)$ is a path in $\mathcal{D}$ and hence in $\overline{\mathcal{D}}$.

To show that $w_{0}, w_{1}, \ldots$ is a good path in $\overline{\mathcal{D}}$, we write

$$
\begin{aligned}
& E \ln \left|w_{t}\right|=E \ln \left|E\left(x_{t} \mid s^{t}\right)\right|=E \ln E\left(\left|x_{t}\right| \mid s^{t}\right) \\
& \geq E\left[E\left(\ln \left|x_{t}\right| \mid s^{t}\right)\right]=E \ln \left|x_{t}\right|=t E \ln \lambda\left(\sigma^{t}\right) .
\end{aligned}
$$

In this chain of relations, we have $E\left|x_{t}\right|<\infty$ and $E|\ln | x_{t}||<\infty$ because $\left|x_{t}\right|$ is essentially bounded and $|\ln | x_{t}||=\left|\ln \lambda\left(\sigma^{1}\right)+\ldots+\ln \lambda\left(\sigma^{t}\right)\right|$, where $\kappa \leq \lambda\left(\sigma^{t}\right) \leq M$ by virtue of Proposition 2. Consequently, we can use Jensen's inequality (Proposition A.2 in the Appendix), which yields the inequality in (6.2). From (6.2), we conclude that $E \ln \left|w_{t}\right|-t E \ln \lambda \geq 0$, and so the path $w_{0}, w_{1}, \ldots$ is good.

From Theorem 5 and Proposition 6, we deduce the following result.

Theorem 6. There exist measurable functions $\mu\left(s^{1}\right)>0$ and $\phi\left(\sigma^{0}\right)>0$ such that $\phi$ satisfies

$$
\kappa \leq \phi\left(\sigma^{0}\right) \leq\left|p\left(\sigma^{1}\right)\right| \quad(\text { a.s. })
$$

and

$$
\lambda\left(\sigma^{1}\right)=\phi\left(\sigma^{1}\right) \mu\left(s^{1}\right) \phi\left(\sigma^{0}\right)^{-1} \text { (a.s.). }
$$

Proof. Consider the good path $w_{0}, w_{1}, \ldots$ considered in Proposition 6 and the random variables $\mu^{t}\left(s^{1}\right), t=1,2, \ldots$, defined by (4.5). By applying Theorem 5 to the dynamical system $\overline{\mathcal{D}}$ and the good path (6.1), we construct a sequence of measurable functions $\xi^{m}\left(s^{1}\right)$ belonging to the convex hull of the set $\left\{\ln \mu^{t}\left(s^{1}\right), t=1,2, \ldots\right\}$ and converging in $L_{1}$ to $\ln \lambda\left(\sigma^{1}\right)+\ln \phi\left(\sigma^{0}\right)-\ln \phi\left(\sigma^{1}\right)$, where $\phi\left(\sigma^{0}\right)$ is measurable 
and satisfies $\kappa \leq \phi\left(\sigma^{0}\right) \leq\left|p\left(\sigma^{1}\right)\right|$. Consequently, there exists a measurable function $\xi\left(s^{1}\right)$ such that $\xi\left(s^{1}\right)=\ln \lambda\left(\sigma^{1}\right)+\ln \phi\left(\sigma^{0}\right)-\ln \phi\left(\sigma^{1}\right)$ (a.s.). We have $e^{\xi\left(s^{1}\right)}=$ $\lambda\left(\sigma^{1}\right) \phi\left(\sigma^{0}\right) \phi\left(\sigma^{1}\right)^{-1} \quad$ (a.s.), and so $\lambda\left(\sigma^{1}\right)=\phi\left(\sigma^{1}\right) e^{\xi\left(s^{1}\right)} \phi\left(\sigma^{0}\right)^{-1}$ (a.s.). Thus it remains to define $\mu\left(s^{1}\right)=e^{\xi\left(s^{1}\right)}$.

Remark. In the above argument we used the following fact. Let $(\Omega, \mathcal{F}, P)$ be a probability space and $\mathcal{G}$ a sub- $\sigma$-algebra of $\mathcal{F}$. If a sequence of $\mathcal{G}$-measurable random variables $\xi^{m}(\omega)$ converges in $L_{1}$ to a random variable $\psi(\omega)$, then there exists a $\mathcal{G}$-measurable random variable $\xi(\omega)$ such that $\psi(\omega)=\xi(\omega)$ (a.s.). To show this consider a subsequence $\xi^{m_{k}}(\omega)$ converging to $\psi(\omega)$ a.s. and observe that the function $\xi(\omega)$ defined as $\lim \xi^{m_{k}}(\omega)$ when this limit exists and is finite, and as 0 otherwise, is $\mathcal{G}$-measurable and satisfies $\xi(\omega)=\psi(\omega)$ (a.s.).

Theorem 7. A von Neumann path in the dynamical system $\mathcal{D}$ exists.

Proof. Let $(x, p, \lambda)$ be an equilibrium in $\overline{\mathcal{D}}$. According to Theorem 6 , the function $\lambda\left(\sigma^{1}\right)$ admits a representation (6.4) where $\mu\left(s^{1}\right)>0$ is a measurable function of $s^{1}$ and $\phi\left(\sigma^{0}\right)>0$ is a measurable function of $\sigma^{0}$ satisfying (6.3). Since $\left(x\left(\sigma^{0}\right), x\left(\sigma^{1}\right) \lambda\left(\sigma^{1}\right)\right) \in C\left(s^{1}\right)$ (a.s.), we have

$$
\left(x\left(\sigma^{0}\right), x\left(\sigma^{1}\right) \phi\left(\sigma^{1}\right) \mu\left(s^{1}\right) \phi\left(\sigma^{0}\right)^{-1}\right) \in C\left(s^{1}\right) \text { (a.s.). }
$$

By using the fact that $C\left(s^{1}\right)$ is a cone, we obtain

$$
\left(x\left(\sigma^{0}\right) \phi\left(\sigma^{0}\right), x\left(\sigma^{1}\right) \phi\left(\sigma^{1}\right) \mu\left(s^{1}\right)\right) \in C\left(s^{1}\right) \text { (a.s.). }
$$

Consequently, $\left(x^{*}\left(\sigma^{0}\right), x^{*}\left(\sigma^{1}\right) \mu\left(s^{1}\right)\right) \in C\left(s^{1}\right)$ (a.s.), where $x^{*}\left(\sigma^{0}\right):=\phi\left(\sigma^{0}\right) x\left(\sigma^{0}\right)$. The functions $x^{*}\left(\sigma^{0}\right)$ and $\mu\left(s^{1}\right) x^{*}\left(\sigma^{1}\right)$ are integrable because $\left|x^{*}\left(\sigma^{0}\right)\right|=\phi\left(\sigma^{0}\right) \leq$ $\left|p\left(\sigma^{1}\right)\right|$ and $\mu\left(s^{1}\right)\left|x^{*}\left(\sigma^{1}\right)\right| \leq M\left|x^{*}\left(\sigma^{0}\right)\right|$. By virtue of Proposition A.4, we have

$$
\left.\left(E\left(x^{*}\left(\sigma^{0}\right) \mid s^{\infty}\right), \mu\left(s^{1}\right) E\left(x^{*}\left(\sigma^{1}\right) \mid s^{\infty}\right)\right)\right) \in C\left(s^{1}\right) \text { (a.s.), }
$$

which yields

$$
\left.\left(E\left(x^{*}\left(\sigma^{0}\right) \mid s^{0}\right), \mu\left(s^{1}\right) E\left(x^{*}\left(\sigma^{1}\right) \mid s^{1}\right)\right)\right) \in C\left(s^{1}\right) \text { (a.s.) }
$$

because $E\left(x^{*}\left(\sigma^{0}\right) \mid s^{\infty}\right)=E\left(x^{*}\left(\sigma^{0}\right) \mid s^{0}\right)$ (a.s.) and $E\left(x^{*}\left(\sigma^{1}\right) \mid s^{\infty}\right)=E\left(x^{*}\left(\sigma^{1}\right) \mid s^{1}\right)$ (a.s.) in view of the non-anticipativity of the process $\left(\sigma_{t}\right)$.

Define $x^{\prime}\left(s^{0}\right):=E\left(x^{*}\left(\sigma^{0}\right) \mid s^{0}\right), \rho\left(s^{0}\right):=\left|x^{\prime}\left(s^{0}\right)\right|=E\left(\left|x^{*}\left(\sigma^{0}\right)\right| \mid s^{0}\right)$ and $\check{x}\left(s^{0}\right):=$ $x^{\prime}\left(s^{0}\right) / \rho\left(s^{0}\right)$. Then

$$
\rho\left(s^{0}\right)=E\left(\left|x^{*}\left(\sigma^{0}\right)\right| \mid s^{0}\right)=E\left(\left|\phi\left(\sigma^{0}\right)\right| \mid s^{0}\right) \geq \kappa,\left|\check{x}\left(s^{0}\right)\right|=1,
$$

and by virtue of (6.5),

$$
\left(\check{x}\left(s^{0}\right), \check{\lambda}\left(s^{1}\right) \check{x}\left(s^{1}\right)\right) \in C\left(s^{1}\right) \quad \text { (a.s.), }
$$

where $\check{\lambda}\left(s^{1}\right):=\rho\left(s^{0}\right)^{-1} \mu\left(s^{1}\right) \rho\left(s^{1}\right)$. By using (6.6), we find $\kappa \leq \rho\left(s^{0}\right) \leq E\left(\left|p\left(\sigma^{1}\right)\right| \mid s^{0}\right)$, and so $E\left|\ln \rho\left(s^{0}\right)\right|<\infty$. It follows from (6.3) and (6.4) that $E\left|\ln \mu\left(s^{1}\right)\right|<\infty$. Thus, $E \ln \check{\lambda}\left(s^{1}\right)=E \ln \mu\left(s^{1}\right)+E \ln \rho\left(s^{1}\right)-E \ln \rho\left(s^{0}\right)=E \ln \mu\left(s^{1}\right)$. Analogously, by using (6.3) and (6.4), we obtain $E \ln \mu\left(s^{1}\right)=E \ln \lambda\left(\sigma^{1}\right)$, which gives $E \ln \check{\lambda}\left(s^{1}\right)=$ $E \ln \lambda\left(\sigma^{1}\right)$.

We have constructed a pair of measurable functions $\check{x}\left(s^{0}\right) \geq 0, \check{\lambda}\left(s^{1}\right)>0$ generating a balanced path in the dynamical system $\mathcal{D}$ (see (6.6) and (6.7)) and such that $E \ln \check{\lambda}\left(s^{1}\right)=E \ln \lambda\left(\sigma^{1}\right)$. By virtue of Proposition $3, E \ln \lambda\left(\sigma^{1}\right) \geq E \ln \lambda^{\prime}\left(\sigma^{1}\right)$ for any pair $x^{\prime}\left(\sigma^{0}\right), \lambda^{\prime}\left(\sigma^{1}\right)$ generating a balanced path in the dynamical system $\overline{\mathcal{D}}$, 
and hence $E \ln \lambda\left(\sigma^{1}\right) \geq E \ln \lambda^{\prime}\left(s^{1}\right)$ for any pair $x^{\prime}\left(s^{0}\right), \lambda^{\prime}\left(s^{1}\right)$ generating a balanced path in $\mathcal{D}$. This means that $\check{x}\left(s^{0}\right), \check{\lambda}\left(s^{1}\right)$ defines a von Neumann path in $\mathcal{D}$.

Theorem 1 is an immediate consequence of Theorems 3 and 7 .

\section{ON THE UNIQUENESS OF AN EQUILIBRIUM GROWTH FACTOR}

Although our assumptions do not guarantee the uniqueness of an equilibrium, it can be shown that an equilibrium growth factor is unique up to homological equivalence.

Theorem 8. Let $(v, p, \lambda)$ and $(w, q, \mu)$ be two equilibria. Then there exists a measurable function $\gamma\left(s^{1}\right)>0$ such that $\mu\left(s^{1}\right)=\gamma\left(s^{1}\right) \lambda\left(s^{1}\right) \gamma\left(s^{0}\right)^{-1}$ (a.s.).

Proof. We will write $w_{0}$ for $w\left(s^{0}\right), w_{1}$ for $w\left(s^{1}\right), \mu_{1}$ for $\mu\left(s^{1}\right)$, etc. From the definition of an equilibrium, we obtain

$$
\mu_{1} \frac{\hat{p}_{1} w_{1}}{p_{1} w_{0}} \leq \lambda_{1}, \lambda_{1} \frac{\hat{q}_{1} v_{1}}{q_{1} v_{0}} \leq \mu_{1} \text { (a.s.) }
$$

where $\hat{p}_{1}=E_{1} p_{2}>0, \hat{q}_{1}=E_{1} q_{2}>0$. Consequently,

$$
\frac{\hat{p}_{1} w_{1}}{p_{1} w_{0}} \leq \frac{\lambda_{1}}{\mu_{1}} \leq \frac{q_{1} v_{0}}{\hat{q}_{1} v_{1}} \text { (a.s.) }
$$

and so

$$
0=E \ln \frac{p_{2} w_{1}}{p_{1} w_{0}} \leq E \ln \frac{\hat{p}_{1} w_{1}}{p_{1} w_{0}} \leq E \ln \frac{\lambda_{1}}{\mu_{1}} \leq E \ln \frac{q_{1} v_{0}}{\hat{q}_{1} v_{1}} \leq E \ln \frac{q_{1} v_{0}}{q_{2} v_{1}}=0,
$$

where the first and the fourth inequalities hold because $E_{1} \ln p_{2} w_{1} \leq \ln \hat{p}_{1} w_{1}$ and $\ln \hat{q}_{1} v_{1} \geq E_{1} \ln q_{2} v_{1}$ (a.s.); see Proposition A.2 (a). From the above relations we get $E \ln \lambda_{1}=E \ln \mu_{1}$,

$$
E \ln E_{1}\left(p_{2} w_{1}\right)=E \ln p_{1} w_{0}=E \ln p_{2} w_{1}=E E_{1}\left(\ln p_{2} w_{1}\right),
$$

and analogously $E \ln E_{1}\left(q_{2} v_{1}\right)=E E_{1}\left(\ln q_{2} v_{1}\right)$. This implies by virtue of Proposition A.2 (b) the existence of measurable functions $\gamma_{1}=\gamma\left(s^{1}\right)>0$ and $\delta_{1}=$ $\delta\left(s^{1}\right)>0$ such that $q_{2} v_{1}=\gamma_{1}$ and $p_{2} w_{1}=\delta_{1}$ (a.s.). Then $q_{1} v_{0}=\gamma_{0}$ and $p_{1} w_{0}=\delta_{0}$ (a.s.) by virtue of stationarity. Furthermore, $\hat{q}_{1} v_{1}=E_{1}\left(q_{2} v_{1}\right)=\gamma_{1}$ and $\hat{p}_{1} w_{1}=E_{1}\left(p_{2} w_{1}\right)=\delta_{1}$ (a.s.). Thus we have $\delta_{1} / \delta_{0} \leq \lambda_{1} / \mu_{1} \leq \gamma_{0} / \gamma_{1}$ (a.s.), which yields $0=E \ln \left(\delta_{1} / \delta_{0}\right) \leq E \ln \left(\lambda_{1} / \mu_{1}\right) \leq E \ln \left(\gamma_{0} / \gamma_{1}\right)=0$. Consequently, $\lambda\left(s^{1}\right) / \mu\left(s^{1}\right)=\gamma\left(s^{0}\right) / \gamma\left(s^{1}\right)$ (a.s.), which proves the theorem.

\section{APPENDIX}

The Appendix contains statements, and in several cases short proofs, of some general facts used in this work. The results are presented in a form convenient for application in this work (sometimes not in their most general form).

Proposition A.1 (Komlós [19]). Let $\xi_{1}, \xi_{2}, \ldots$ be random vectors in $\mathbb{R}^{n}$ with $\sup E\left|\xi_{N}\right|<\infty$. Then there exists a random vector $\xi$ and natural numbers $N_{1}<$ $N_{2}<\ldots$ such that

$$
m^{-1}\left(\xi_{N_{1}}+\ldots+\xi_{N_{m}}\right) \rightarrow \xi(\text { a.s. }),
$$

and (A.1) holds for any subsequence of $\left\{N_{m}\right\}$.

Let $(\Omega, \mathcal{F}, P)$ be a probability space and $\mathcal{G}$ a sub- $\sigma$-algebra of $\mathcal{F}$. We write $E^{\mathcal{G}}$ and $P^{\mathcal{G}}$ for the conditional expectation and the conditional probability given $\mathcal{G}$. 
Proposition A.2. Let $f(x)$ be a Borel function defined on an interval $(a, b)(-\infty \leq$ $a<b \leq+\infty)$ and let $\xi(\omega)$ be a random variable taking values in $(a, b)$ such that $E|f(\xi)|<\infty$ and $E|\xi|<\infty$ (a.s.). (a) If $f(x)$ is concave, then $f\left(E^{\mathcal{G}} \xi\right) \geq E^{\mathcal{G}} f(\xi)$ (a.s.). (b) If $f(x)$ is strictly concave and $f\left(E^{\mathcal{G}} \xi\right)=E^{\mathcal{G}} f(\xi)$ (a.s.), then $\xi$ coincides a.s. with a $\mathcal{G}$-measurable random variable.

Assertion (a) is a version of Jensen's inequality for conditional expectations; see, e.g., Loève [20, Section 9.3]. Assertion (b) can be proved by modifying the argument used for proving (a) in [20, Section 9.3].

Denote by $\mathcal{E}$ the set of random variables $\xi$ such that $E e^{\xi}<\infty$. Note that this set is convex because the function $e^{x}$ is convex.

Proposition A.3. The operator $\xi \mapsto \ln E^{\mathcal{G}} e^{\xi}$ is convex on the set $\mathcal{E}$ and maps this set into itself.

The second assertion follows from the relations $E \exp \left(\ln E^{\mathcal{G}} e^{\xi}\right)=E\left(E^{\mathcal{G}} e^{\xi}\right)=$ $E e^{\xi}$. Let $r \in(0,1)$ be a number and $\xi, \eta$ two random variables such that $E e^{\xi}<\infty$ and $E e^{\eta}<\infty$. The convexity of the operator under consideration means that $\ln E^{\mathcal{G}} e^{r \xi+(1-r) \eta} \leq r \ln E^{\mathcal{G}} e^{\xi}+(1-r) \ln E^{\mathcal{G}} e^{\eta}$ or, equivalently, $E^{\mathcal{G}} e^{r \xi+(1-r) \eta} \leq$ $\left(E^{\mathcal{G}} e^{\xi}\right)^{r}\left(E^{\mathcal{G}} e^{\eta}\right)^{1-r}$. By setting $\alpha=e^{r \xi}$ and $\beta=e^{(1-r) \eta}$, we rewrite this inequality as $E^{\mathcal{G}} \alpha \beta \leq\left[E^{\mathcal{G}}\left(\alpha^{1 / r}\right)\right]^{r}\left[E^{\mathcal{G}} \beta^{1 /(1-r)}\right]^{(1-r)}$, which is the Hölder inequality for conditional expectations (see, e.g., [20, Section 25.1]).

Let $C(\omega) \subseteq \mathbb{R}^{n}$ be a non-empty closed set $\mathcal{G}$-measurably depending on $\omega$.

Proposition A.4. If $C(\omega)$ is convex and if $\xi(\omega)$ is a random vector such that $\xi(\omega) \in C(\omega)$ (a.s.) and $E|\xi(\omega)|<\infty$, then $E(\xi(\omega) \mid \mathcal{G}) \in C(\omega)$ (a.s.).

For a proof see [1, Appendix II, Lemma 1].

Proposition A.5. Let $f(\omega, x)$ be a real-valued jointly measurable function of $\omega \in$ $\Omega$ and $x \in \mathbb{R}^{n}$ such that for $x \in C(\omega)$ we have $|f(\omega, x)| \leq K(\omega)$, where $E K(\omega)<\infty$. Let $\bar{\xi}(\omega)$ be a measurable vector function such that $\bar{\xi}(\omega) \in C(\omega)$ (a.s.). Then the following assertions are equivalent. (i) The inequality $\operatorname{Ef}(\omega, \xi(\omega)) \leq E f(\omega, \bar{\xi}(\omega))$ holds for all measurable functions $\xi(\omega)$ satisfying $\xi(\omega) \in C(\omega)$ (a.s.). (ii) With probability one,

$$
f(\omega, \bar{\xi}(\omega))=\max _{x \in C(\omega)} f(\omega, x) .
$$

The result can be obtained by using a measurable selection theorem (see [1, Appendix I]).

Let $D_{1}$ and $D_{2}$ be Banach spaces, $W$ a convex subset of $D_{1}, F: W \rightarrow(-\infty,+\infty)$ a concave functional and $B: D_{1} \rightarrow D_{2}$ a continuous linear operator. Assume that the functional $F(w)$ attains its maximum on the set $\{w \in W: B w=0\}$ at some point $\bar{w}$.

Proposition A.6. Let the following conditions hold. (a) The set $\{w \in W: B w=$ $0\}$ contains a point belonging to the interior of the set $W$. (b) The image of $D_{1}$ under the mapping $B: D_{1} \rightarrow D_{2}$ is a closed subspace of $D_{2}$. (c) The functional $F(\cdot)$ is bounded below on some open subset of $W$. Then there exists a continuous linear functional $\pi$ on the space $D_{2}$ such that $F(w)+\langle\pi, B w\rangle \leq F(\bar{w})$ for each $w \in W$.

This result is an infinite-dimensional version of the Kuhn-Tucker theorem for concave optimization problems with linear equality constraints. The functional $\pi$ 
is a "Lagrange multiplier" associated with the constraint $B w=0$. Proposition A.6 is a consequence of Proposition 1 in [10].

Let $L_{\infty}=L_{\infty}\left(\Omega, \mathcal{F}, P, \mathbb{R}^{n}\right)$ be the Banach space of essentially bounded random vectors with values in $\mathbb{R}^{n}$ with the norm $\|\cdot\|_{\infty}$, and let $L_{\infty}^{*}$ be the dual of $L_{\infty}$.

Proposition A.7. Any functional $\pi \in L_{\infty}^{*}$ can be decomposed into the sum $\pi=$ $\pi^{a}+\pi^{s}$ of two functionals $\pi^{a} \in L_{\infty}^{*}$ and $\pi^{s} \in L_{\infty}^{*}$ possessing the following properties:

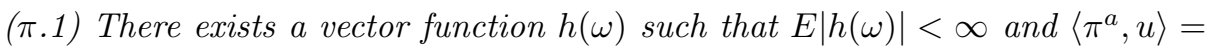
$\operatorname{Eh}(\omega) u(\omega)$ for all $u \in L_{\infty}$.

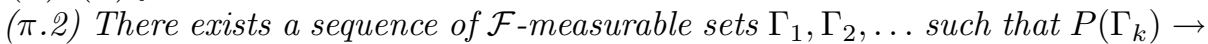
0 and $\left\langle\pi^{s}, u\right\rangle=\left\langle\pi^{s}, \mathbf{1}_{\Gamma_{k}} u\right\rangle$ for all $k=1,2, \ldots$ and $u \in L_{\infty}$.

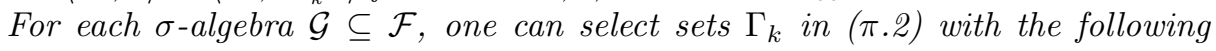
additional property:

$$
\left\|\mathbf{1}_{\Omega \backslash \Gamma_{k}} P^{\mathcal{G}}\left(\Gamma_{k}\right)\right\|_{\infty} \rightarrow 0 .
$$

The main content of the above proposition, providing a decomposition of any functional $\pi \in L_{\infty}^{*}$ into a sum $\pi^{a}+\pi^{s}$ of an absolutely continuous and singular ones, is the Yosida-Hewitt theorem [30]. To obtain the additional property (A.3), we may assume without loss of generality that the sets in $\Gamma_{k}$ in $(\pi .2)$ satisfy $P\left(\Gamma_{k}\right)<k^{-2}$. Define $\Gamma_{k}^{\prime}:=\left\{\omega: P^{\mathcal{G}}\left(\Gamma_{k}\right) \geq k^{-1}\right\}$ and $\Delta_{k}:=\Gamma_{k} \cup \Gamma_{k}^{\prime}$. Then $\left\langle\pi^{s}, \mathbf{1}_{\Delta_{k}} u\right\rangle=\left\langle\pi^{s}, \mathbf{1}_{\Gamma_{k}} \mathbf{1}_{\Delta_{k}} u\right\rangle=\left\langle\pi^{s}, \mathbf{1}_{\Gamma_{k}} u\right\rangle=\left\langle\pi^{s}, u\right\rangle$ because $\Gamma_{k} \subseteq \Delta_{k}$. Further, $P\left(\Gamma_{k}^{\prime}\right) \leq k E\left[P^{\mathcal{G}}\left(\Gamma_{k}\right)\right]=k P\left(\Gamma_{k}\right) \leq k^{-1}$, and so $P\left(\Delta_{k}\right) \leq k^{-2}+k^{-1} \rightarrow 0$. Finally, $\mathbf{1}_{\Omega \backslash \Delta_{k}} P^{\mathcal{G}}\left(\Delta_{k}\right) \leq \mathbf{1}_{\Omega \backslash \Delta_{k}} P^{\mathcal{G}}\left(\Gamma_{k}+\Gamma_{k}^{\prime}\right)=\mathbf{1}_{\Omega \backslash \Gamma_{k}} \mathbf{1}_{\Omega \backslash \Gamma_{k}^{\prime}}\left[P^{\mathcal{G}}\left(\Gamma_{k}\right)+P^{\mathcal{G}}\left(\Gamma_{k}^{\prime}\right)\right] \leq k^{-1}$, which shows that the sets $\Delta_{k}$ possess the properties listed in $(\pi .2)$ and (A.3).

\section{REFERENCES}

[1] V. I. Arkin and I. V. Evstigneev. Stochastic models of control and economic dynamics. Academic Press, 1987.

[2] L. Arnold, I. V. Evstigneev and V. M. Gundlach. Convex-valued random dynamical systems: A variational principle for equilibrium states. Random Operators and Stochastic Equations 7 (1999), 23-38. MR1677758 (2000j:60078)

[3] L. Arnold, V. M. Gundlach and L. Demetrius. Evolutionary formalism for products of positive random matrices. Annals of Applied Probability 4 (1994), 859-901. MR1284989 (95h:28028)

[4] M. A. H. Dempster, I. V. Evstigneev and M. I. Taksar. Asset pricing and hedging in financial markets with transaction costs: An approach based on the von Neumann-Gale model. Annals of Finance 2 (2006), 327-355.

[5] A. Dvoretzky, A. Wald, J. Wolfowitz. Elimination of randomization in certain problems of statistics and of the theory of games. Proc. Nat. Acad. Sci. 36 (1950), 256-260. MR0035979 $(12: 40 \mathrm{c})$

[6] E. B. Dynkin. Some probability models for a developing economy. Soviet Mathematics Doklady 12 (1971), 1422-1425.

[7] E. B. Dynkin. Stochastic concave dynamic programming. Math. USSR Sbornik 16 (1972), 501-515.

[8] E. B. Dynkin and A. A. Yushkevich. Controlled Markov processes and their applications. Springer, 1979. MR554083 (80k:90037)

[9] I. V. Evstigneev. Positive matrix-valued cocycles over dynamical systems. Uspekhi Matem. Nauk (Russ. Math. Surveys) 29 (1974), 219-220. (In Russian.) MR0396906 (53:766)

[10] I. V. Evstigneev and S. D. Flåm. Stochastic programming: Non-anticipativity and Lagrange multipliers. In: Encyclopedia of Optimization, Kluwer Academic Publishers, Vol. 4, 2001, pp. $332-338$.

[11] I. V. Evstigneev and K. R. Schenk-Hoppé. The von Neumann-Gale growth model and its stochastic generalization. In: R. Dana, C. Le Van, T. Mitra, K. Nishimura (eds.), Handbook on Optimal Growth, Vol. I, Chapter 2, Springer, 2006, pp. 337-383. 
[12] I. V. Evstigneev and K. R. Schenk-Hoppé. Pure and randomized equilibria in the stochastic von Neumann-Gale model. Journal of Mathematical Economics 43 (2007), 871-887. MR2341683

[13] I. V. Evstigneev and M. I. Taksar. Rapid growth paths in convex-valued random dynamical systems. Stochastics and Dynamics 1 (2001), 493-509. MR1875064 (2002i:60082)

[14] D. Gale. A closed linear model of production. In: H. W. Kuhn et al. (eds.), Linear Inequalities and Related Systems, Princeton University Press, 1956, pp. 285-303. MR0085961 (19:105d)

[15] D. Gale. A mathematical theory of optimal economic development. Bull. Amer. Math. Soc. 74 (1968), 207-223. MR0221835 (36:4887)

[16] D. Gale. A note on the nonexistence of optimal price vectors in the general balanced-growth model of Gale: Comment. Econometrica 40 (1972), 391-392.

[17] J. Hulsmann and V. Steinmetz. A note on the nonexistence of optimal price vectors in the general balanced-growth model of Gale. Econometrica 40 (1972), 387-389. MR0378726 (51:14892)

[18] Y. Kifer. Perron-Frobenius theorem, large deviations, and random perturbations in random environments. Mathematische Zeitschrift 222 (1996), 677-698. MR1406273 (97f:60131)

[19] J. Komlós. A generalization of a problem of Steinhaus. Acta Math. Acad. Sci. Hungar. 18 (1967), 217-229. MR0210177 (35:1071)

[20] M. Loève. Probability theory. Van Nostrand, 1960. MR0203748 (34:3596)

[21] V. L. Makarov and A. M. Rubinov. Mathematical theory of economic dynamics and equilibria. Springer, 1977. MR0439072 (55:11973)

[22] H. Nikaido. Convex structures and economic theory. Academic Press, 1968. MR0277233 (43:2970)

[23] R. Radner. Balanced stochastic growth at the maximum rate. In: Contributions to the von Neumann Growth Model (Proc. Conf., Inst. Adv. Studies, Vienna, 1970), Z. Nationalökonomie, Suppl. No. 1, 1971, pp. 39-53. MR0325117 (48:3466)

[24] R. Radner. Optimal steady-state behavior of an economy with stochastic production and resources. In: R. H. Day and S. M. Robinson (eds.), Mathematical Topics in Economic Theory and Computation, SIAM, 1972, pp. 99-112. MR0406415 (53:10202)

[25] R. Radner. Optimal stationary consumption with stochastic production and resources. $J$. Econ. Theory 6 (1973), 68-90. MR0452560 (56:10839)

[26] R. Radner. Equilibrium under uncertainty. In: K. J. Arrow and M. D. Intrilligator (eds.), Handbook of Mathematical Economics, North-Holland, 1982, pp. 923-1006.

[27] R. T. Rockafellar. Monotone processes of convex and concave type. Memoirs of the Amer. Math. Soc. 77, 1967. MR0225231 (37:825)

[28] R. T. Rockafellar and R. J.-B. Wets. Nonanticipativity and $L^{1}$-martingales in stochastic optimization problems. In: R.J.-B. Wets (ed.), Stochastic Systems: Modeling, Identification and Optimization II, Math. Programming Study 6 (1976), 170-187. MR0462590 (57:2563)

[29] J. von Neumann. Über ein ökonomisches Gleichungssystem und eine Verallgemeinerung des Brouwerschen Fixpunktsatzes. In: Ergebnisse eines Mathematischen Kolloquiums, No. 8, 1935-1936, Franz-Deuticke, 1937, pp. 73-83. [Translated: A model of general economic equilibrium, Review of Economic Studies 13 (1945-1946), 1-9.]

[30] K. Yosida and E. Hewitt. Finitely additive measures. Trans. Amer. Math. Soc. 72 (1952), 46-66. MR0045194 (13:543b)

Economics Department, University of Manchester, Oxford Road, Manchester, M13 9PL, United Kingdom

E-mail address: igor.evstigneev@manchester.ac.uk

School of Mathematics and Leeds University Business School, Leeds University, LeEds LS2 9JT, UNITED KINGDOM

E-mail address: K.R.Schenk-Hoppe@leeds.ac.uk 